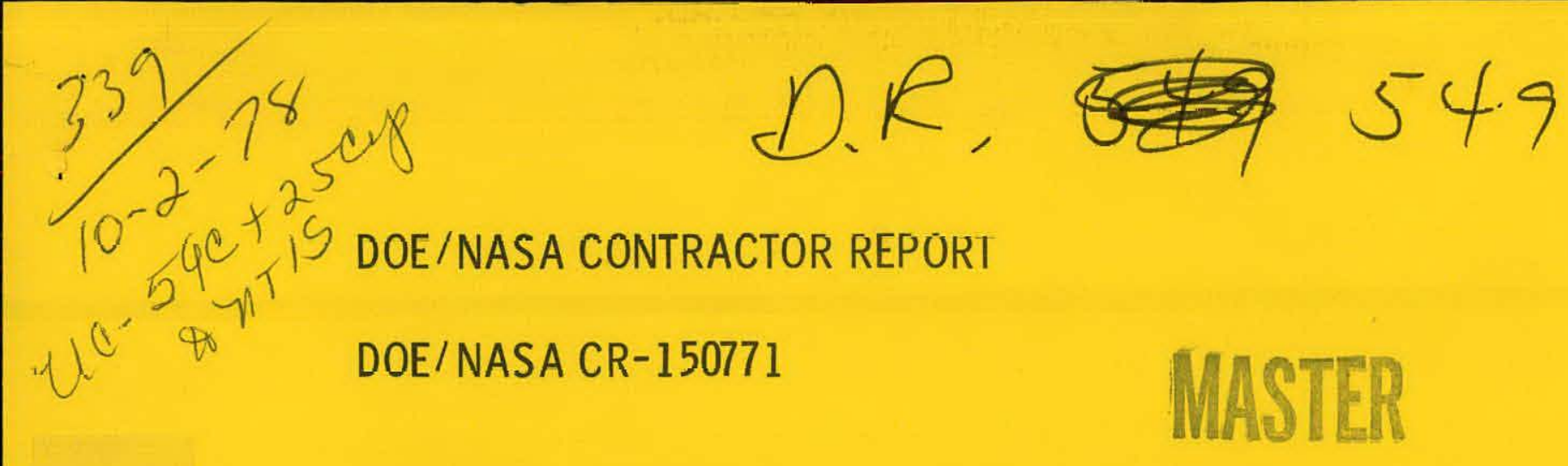

\title{
SOLAR CONTROL DESIGN PACKAGE
}

Prepared from documents provided by

Solar Control Corporation

5595 Arapahoe Road

Boulder, Colorado 80302

Under Contract NAS8-32258 with

\section{NOTICE}

PORTIONS OF THIS REPORT ARE ILLEGIBLE. It has been repragiced from the best available copy to permit the broadest possible avail. ability.

National Aeronautics and Space Administration

George C. Marshall Space Flight Center, Alabama 35812

For the U. S. Department of Energy

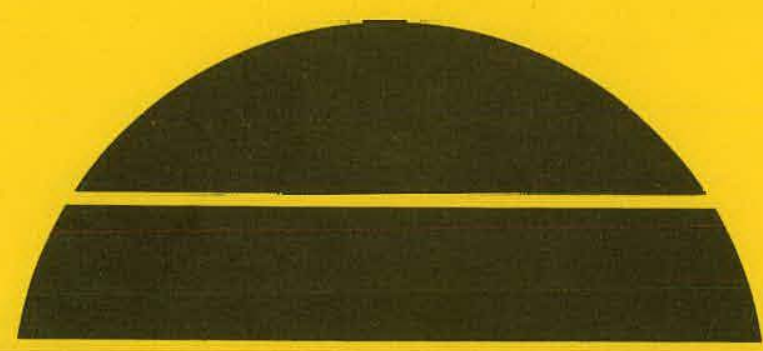

\section{U.S. Department of Energy}




\section{DISCLAIMER}

This report was prepared as an account of work sponsored by an agency of the United States Government. Neither the United States Government nor any agency Thereof, nor any of their employees, makes any warranty, express or implied, or assumes any legal liability or responsibility for the accuracy, completeness, or usefulness of any information, apparatus, product, or process disclosed, or represents that its use would not infringe privately owned rights. Reference herein to any specific commercial product, process, or service by trade name, trademark, manufacturer, or otherwise does not necessarily constitute or imply its endorsement, recommendation, or favoring by the United States Government or any agency thereof. The views and opinions of authors expressed herein do not necessarily state or reflect those of the United States Government or any agency thereof. 


\section{DISCLAIMER}

Portions of this document may be illegible in electronic image products. Images are produced from the best available original document. 
NOTICE

This report was prepared to document work sponsored by the United States Government. Ne1ther the United States nor 1ts agents the United States Department of Energy, the United States National Aeronautics and Space Administration, nor any federal employees, nor any of their contractors, subcontractors or their employees, make any warranty, express or implied, or assume any legal lisbility or responsibility for the accuracy, completensss, or usefulness of any information, apparatus, product or process disclosed, or represent that 1 ts use would not infringe privately owned rights. 
TECHNICAL REPORT ST ANDARD TITLE PAGE

1. REPORT NO.
DOE/NASA CR-150771

4. TITLE AND SUBTITLE

Solar Control Design Package

7. AUTHOR(S)

2. GOVERNMENT ACCESSION NO.
3. RECIPIENT'S CATALOG NO.

5. REPORT DATE

August 1978

6. PERFORMING ORGANIZATION CCIDE

8. PERFORMING ORGANIZATION REPORT \#

9. PERFORMING ORGANIZATION NAME AND ADDRESS

Solar Control Corporation

5595 Arapahoe Road

Boulder, CO 80302

12. SPONSORING AGENCY NAME AND ADDRESS

National Aeronautics and Space Administration

Washington, D. C. 20546

10. WORK UNIT. NO.

11. CONTRACT OR GRANT NO.

NAS8-32258

13. TYPE OF REPOR'I \& PERIOD COVERED

Contractor Report

14. SPONSORING AGENCY CODE

15. SUPPLEMENTARY NOTES

This work was done under the technical management of $\mathrm{Mr}$. James Hankins, George C. Marshall Space Flight Center, Alabama.

16. ABSTRACT

This report contains information used in the evaluation of design of Solar Control's solar heating and cooling system controller and the Solarstat. Some of the information includes system performance specifications, design data brochures, and detailed design drawings.

Slight modifications have been made for.clarification only.
18. DISTRIBUTION STATEMENT

\section{Unclassified-Unlimited}

WILLIAM A. BROOKSBANK, JR.
Mgr, Solar Heating and Cooling Project Office
19. SECURITY CLASSIF. (of this repart)

Unclassified
20. SECURITY CLASSIF. (ot thls page)

Unclassified
56
NTIS 
THIS PAGE

WAS INTENTIONALLY

LEFT BLANK 
PORTIONS OF THIS REPORT ARE ILLEGIBLE. It

has been reproduced from the best available

copy to permit the broadest possible availe Motrice

ability.

TABLE OF CONTENTS

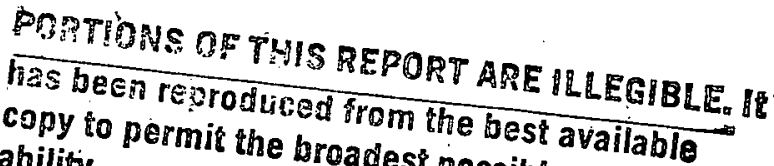
ability.

SUBSYSTEM PERFORMANCE SPECIFICATION

MT ONEY

1.0 Introduction

2.0 Applicable Documents 1

3. 0 Application of Interim Performance Criteria 1

4. 0 Deviation from Interim Performance Criteria 1

5. 0 Government Furnished Property 2

6. 0 Government Directed Requirements 2

7. 0 Subsystem General Specifications and Features 2

DESIGN DATA BROCHURE - Controller 5

Drawings . . 9

DESIGN DATA BROCHURE - Solarstat 30

$\begin{array}{ll}\text { Drawings } & 32\end{array}$

NOTICE MN ONLY

PORTIONS OF THIS PEPORT ARE ILLEGIBLE. It

has been reoroduced from the best available

copy to permit the broadest possible availe

ability. 


\section{SUBSYSTEM PERFGRMANCE SPFCIFICATION}

Specification No.SHC-3OH4

Page Date

$3-9-77$

Page of

1.0 INTRODUCTION

This $P \in r$ formance Specification establishes the requirements for the design and performance of the subsystem. It designates the Interim Performance Criteria applicable to the subsystem and defines the deviations.

$2.0 \quad$ APPLICABLE DOCUMENTS

2.1 Government Documents

See Apendix F

2.2 Contractor Documents

-Application Note 74-116 for Solar Heating and Cooling, 1974 .

-Zia Four Mode Controllers - 非5-140 and \#75-160, 1975.

-Zia Modular Controllers - 非5-170 Series, 1975.

-Zia 非5-150 Solar Air Handling Moduls (SAM), 1975.

\subsection{Other Documents}

-Proceedings of the Solar Heating and Cooling For Buildings

Workshop, Part I: Technical Sessions, March 21 and 22, NSF/RA/N73-004.

-Solar Heating Handbook For Los Alamos, LA-5967 May 1975.

3.0 APPLICATION OF INTERIM PERFORMANCE CRITERIA

The application of each paragraph of the Interim Performance Criteria to the subsystem is provided in the following table, Number 1.

4.0 DEVIATIONS FROM INTERIM PERFORMANCE CRITERIA

None 

of

5.0 GCVSRAAENT FURNISHED PROPERTY

None

6.0 COVERNMENT DIRECTED REQUIREMENTS

7.0 SUBSYSTEM GENERAL SPECIFICATION AND FEATURES

The following. page delineates the general specifications and features of the Series 75-170, Modular-Control Subsystem. 


\section{Modular Controller Sorses 75-170 \\ Table I}

Residential subsystems: Interim Performance Criteria Summary The following. Interim Performance Criterla paragraphs are applicable:

\subsection{Control}

1.7.1 Installation and Maintenance

1.7.3 Inhabited Space Temperature

2.5 Subsystem Isolation

2.5.1 Shutdown in Multi-Family Housing

4.1 Plumbing and Flectrical Installation

4.1.2 Electrical Codes

4.2 Fall-safe Controls

4.2.1 System Fallure Prevention

5.? Temperature and Fressure Resistance

5.2.1 Thermal Ieeradation

6.1 Accessibility for Maintenance

6.1.1 Access for System Maintenance

6.1.2 Access for System Monitoring

6.2 Installation, Operation, and Maintenance Manual

6.2.1 Installation Instructions

6.2.2 Maintenance and Operation Instructions

6.2.3 Maintenance Plan

6.2.4 Peplacement Parts

6.3 Repair and Service Personnel

6.3 .1 !'aintenance of $\mathrm{H}$ and $\mathrm{HC}$ systems

11.3.1 Material Comotibility 
General Specifications:

1. Input Voltage:

2. Input Power:

3. Outputs

4. Output Current

5. Output Voltage

6. Sensors

7. Differential Turnoff offset

8. Differential Turnon Offset

9. Operating Temperature Range 10. Tracking Accuracy

11. Isolation Protection

12. Size

13. Weight

14. Multichannel Priority

15. Mounting

16. Collector freeze protect (differential signal "on" typically; remote signal also available)

17. Manual "on" Button

18. Operating Light

19. Distribution Channel and Auxiliary Heater Control

20. Multi-Tank Control
Either 120 VAC \pm 10 V or 24 VAC $\pm 4 \mathrm{~V}$

3 watts (no-load)

DPDT (3PDT available)

10 amp (resistive) at 28 VDC or 120 VAC (higher.ratings availabie)

Either 120 VAC or 24 VAC Matched thermistors, ( ${ }^{\circ} \mathrm{C}$ over range from $0^{\circ} \mathrm{C}$ to $\left.+100^{\circ} \mathrm{C}\right)$ in a metal housing with high temperature teflon leads

Typically $4^{\circ} \mathrm{F} \pm 2^{\circ}$ อ $100^{\circ} \mathrm{F}$ (can be custom adjuśted by a resistor change)

Typically $20^{\circ} \mathrm{F}+2^{\circ}$ @ $100^{\circ} \mathrm{F}$ (can be custom adjusted by a resistor change)

Controller chassis: ${ }^{\circ} 0^{\circ} \mathrm{F}$ to $140^{\circ} \mathrm{F} \mid$ $\pm 5 \mathrm{~F}^{\circ}$ over entire operating range

$1500 \mathrm{~V}$

Basic Module about 4" $\times 4 " \times 3 \frac{1}{2} "$

Less than 2 Ib.

24 VAC interconnect logic

Mount on "J" box or on wall or pump hausing

At $40^{\circ} \mathrm{F} \pm 5^{\circ}$
100,000 hr. LED illuminates to indicate operation

For 75-172, 75-173 configuration

For 75-175 configuration 

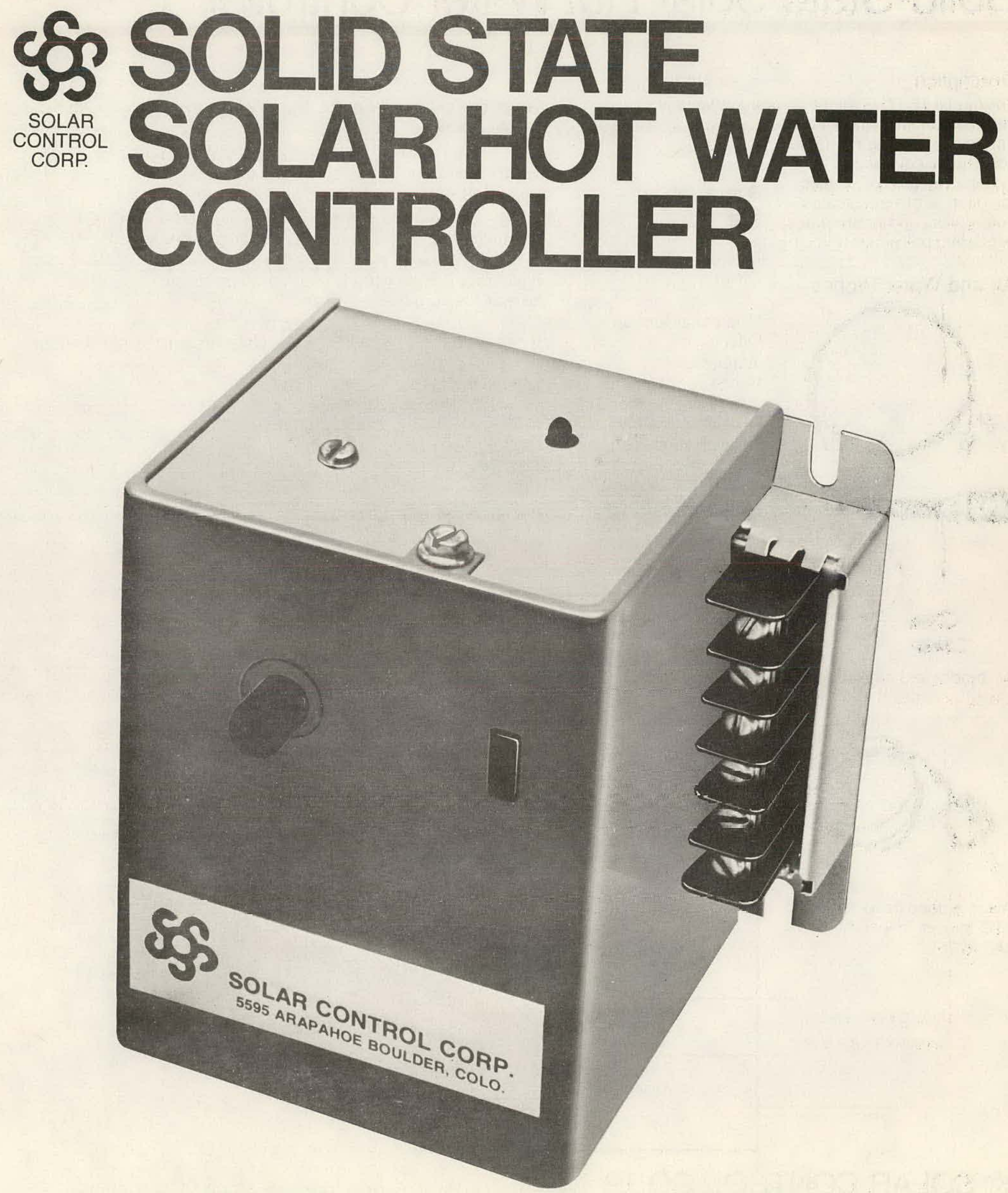


\section{Model 77-171}

\section{Solid-State Solar Hot Water Controller}

\section{Description}

The model $77-171$ is a solidstate differential thermostat capable of fully controlling a solar hot water heating system. The unit is designed for a lifetime of maintenance free service, and incorporates freeze and boil protect circuitry.

\section{Air and Water Probes}
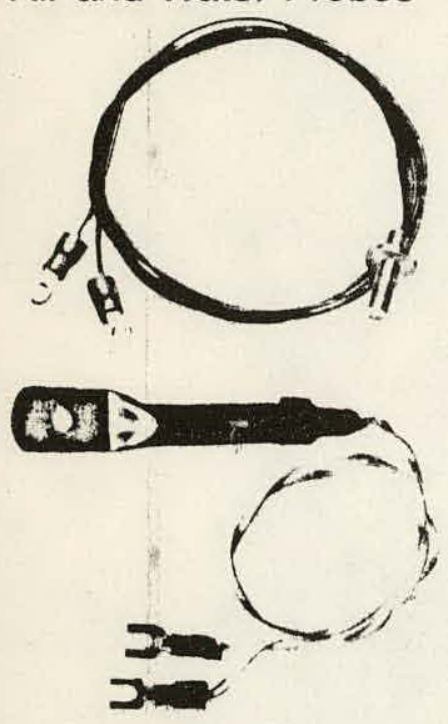

Air probes are available for clamp or screw mounting.

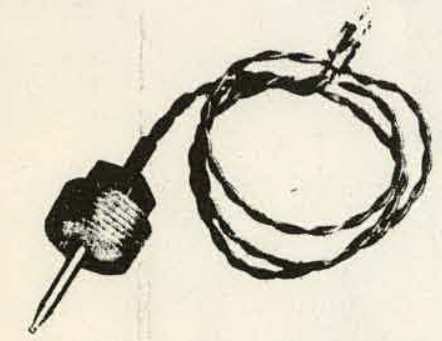

Water probes have $1 / 2^{\prime \prime}$ NPC thread, in lengths from $23 / 4^{\prime \prime}$ to $28^{\prime \prime}$.

\section{Specifications}

Input Voltage ........... Either $120 \mathrm{VAC} \pm 10 \mathrm{~V}$ or $24 \mathrm{VAC} \pm 4 \mathrm{~V}$

Input Power .......... 3 watts, no load.

Control Relay .......... SPDT, 10A. (resistive) at 28 VDC or 120 VAC. Higher ratings available.

Output Voltage .......... Either 120 VAC or 24 VAC.

Sensors ............. Matched thermistors, $\left( \pm 1^{\circ} \mathrm{C}\right.$ over range from $0^{\circ} \mathrm{C}$ to $\left.+100^{\circ} \mathrm{C}\right)$

in a metal housing, with high temperature teflon leads.

Differential Turn-off

Offset Typically $4^{\circ} \mathrm{F} \pm 2^{\circ} @ 100^{\circ} \mathrm{F}$, (can be adjusted by a resistor change).

Differential Turn-on

Offset ............... Typically $20^{\circ} \mathrm{F} \pm 2^{\circ} @ 100^{\circ} \mathrm{F}$, (can be adjusted by a resistor change).

Temperature

Range .............. Controller chassis: $-40^{\circ} \mathrm{F}$ to $140^{\circ} \mathrm{F}$

Sensors: $-40^{\circ} \mathrm{F}$ to $+300^{\circ} \mathrm{F}$

Tracking Accuracy $\ldots \ldots \ldots \pm 5^{\circ} \mathrm{F}$ over entire operating range.

Isolation Protection....... $1500 \mathrm{~V}$

Size ................. $4^{\prime \prime} \times 4^{\prime \prime} \times 3 \frac{1 / 2 "}{2}$

Weight ............... 2 lbs.

Mounting ............. Mounts on 4 " $\times 4$ " "J" box.

Wiring Diagram

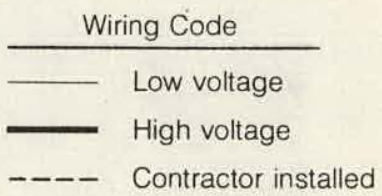

Relay closes when collector temperature is higher than storage temperature.

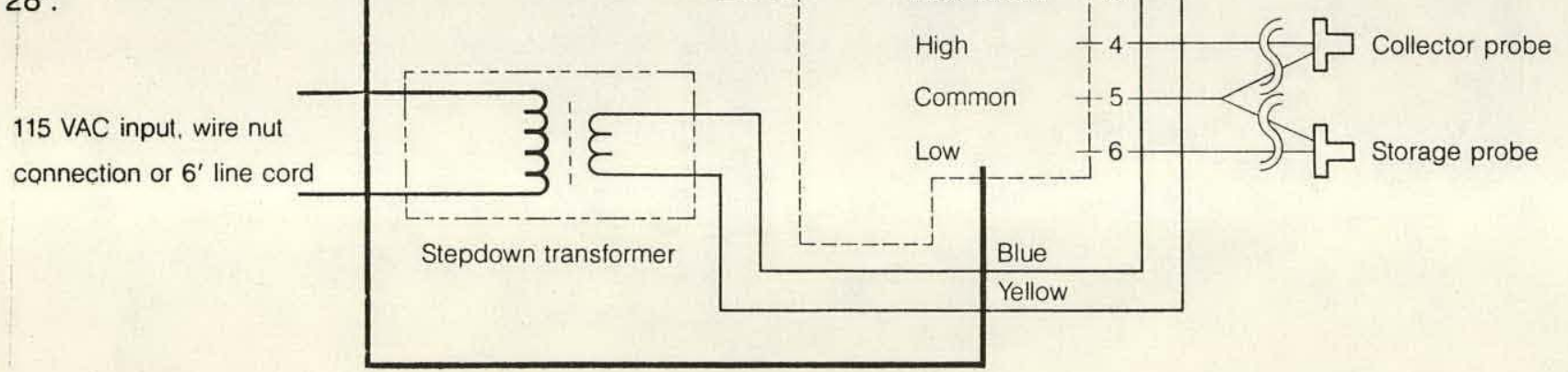

๘্ডో SOLAR CONTROL CORP. 5595 Arapahoe Road, Boulder, Colorado 80302 303/449-9180 


\author{
Contact Solar Control Corporation \\ 5595 Arapahoe Road \\ Boulder, CO 80302 \\ for information on drawings which were unavailable for \\ publishing.
}


THIS PAGE

WAS INTENTIONALLY

LEFT BLANK 
HAT IA SSOCIATES INC.

10101

i

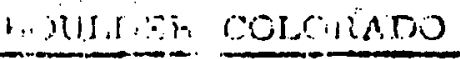

vown 052000

SHEE

1) $\ddot{T}:$

(1) 


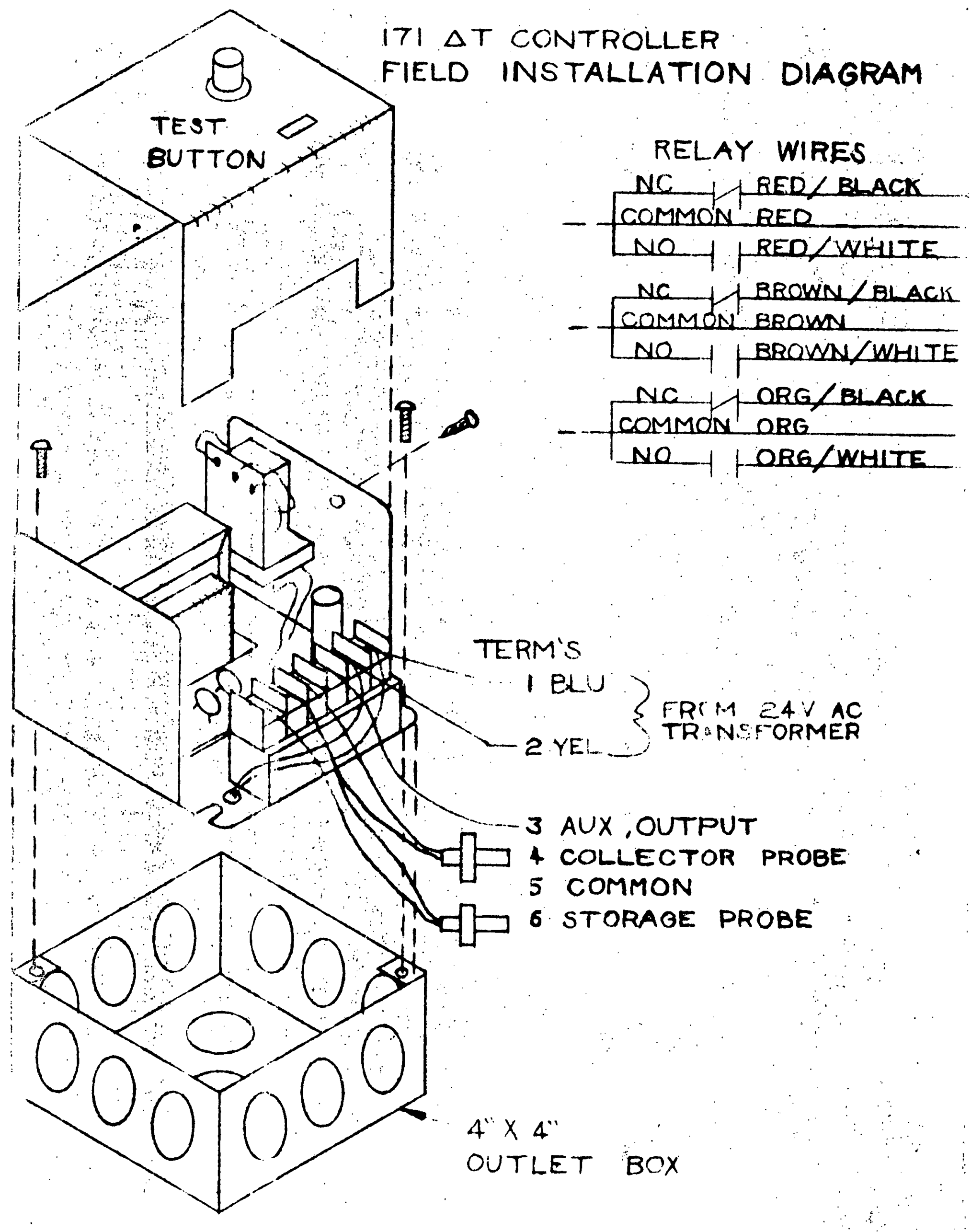

12 


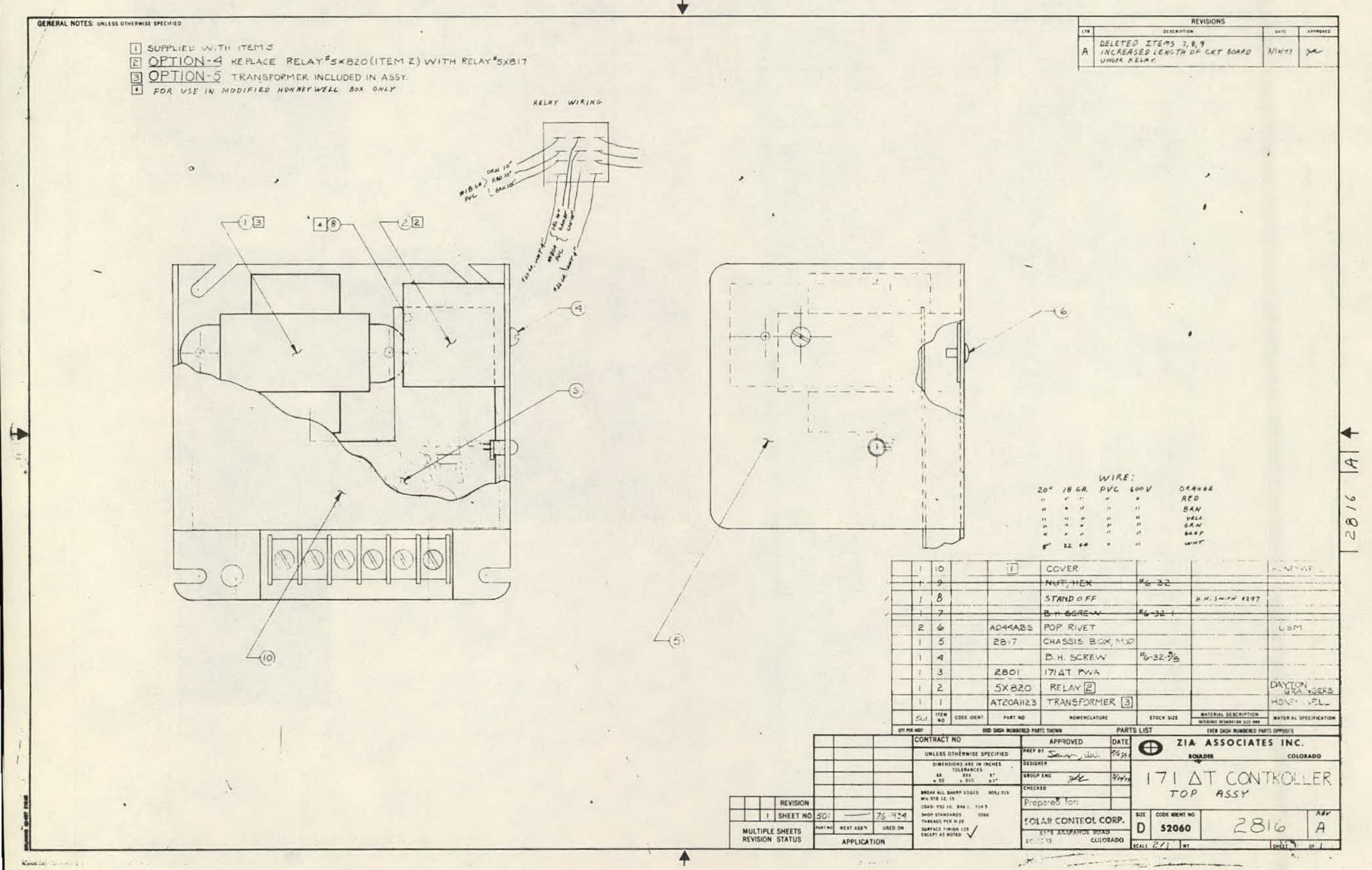




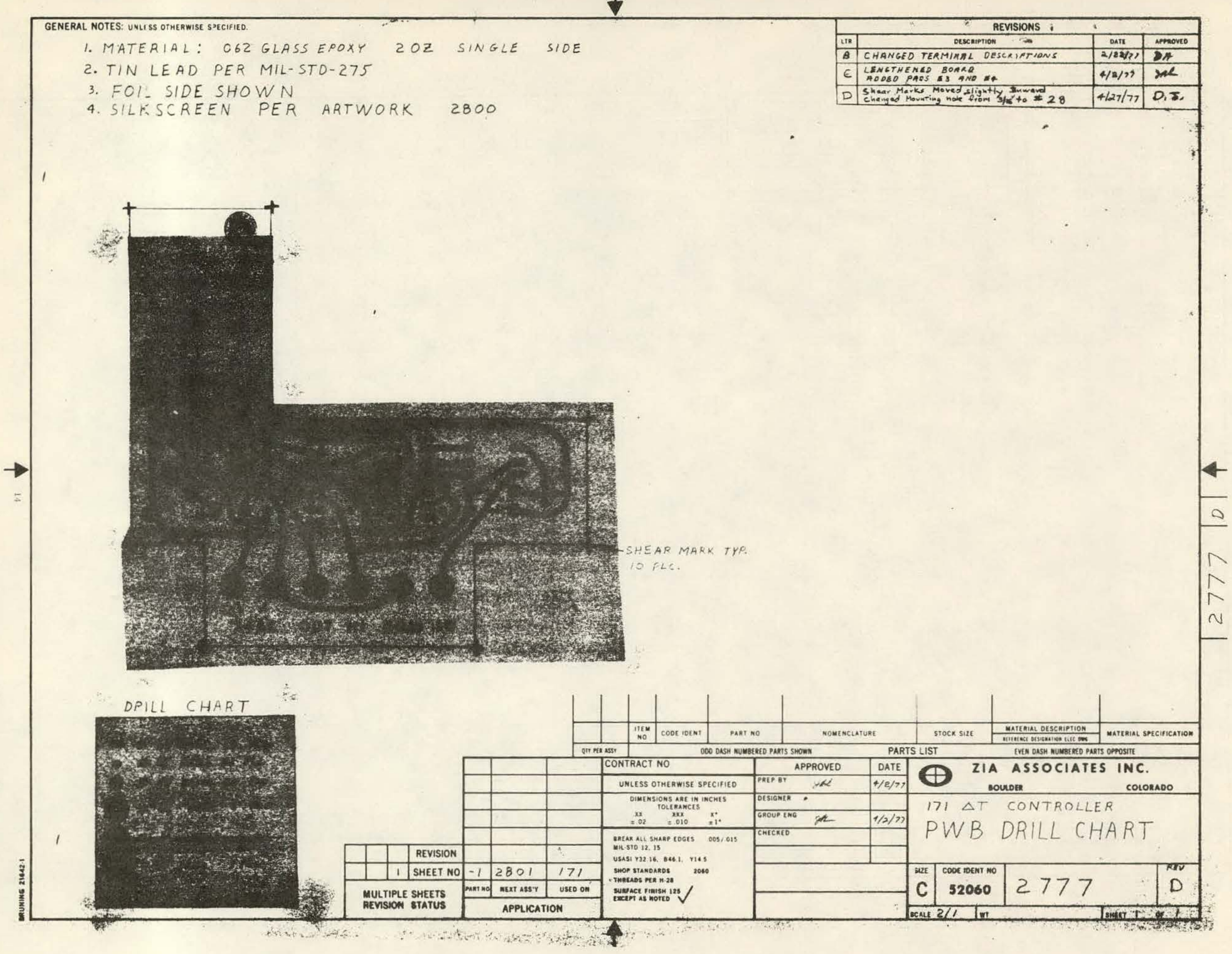




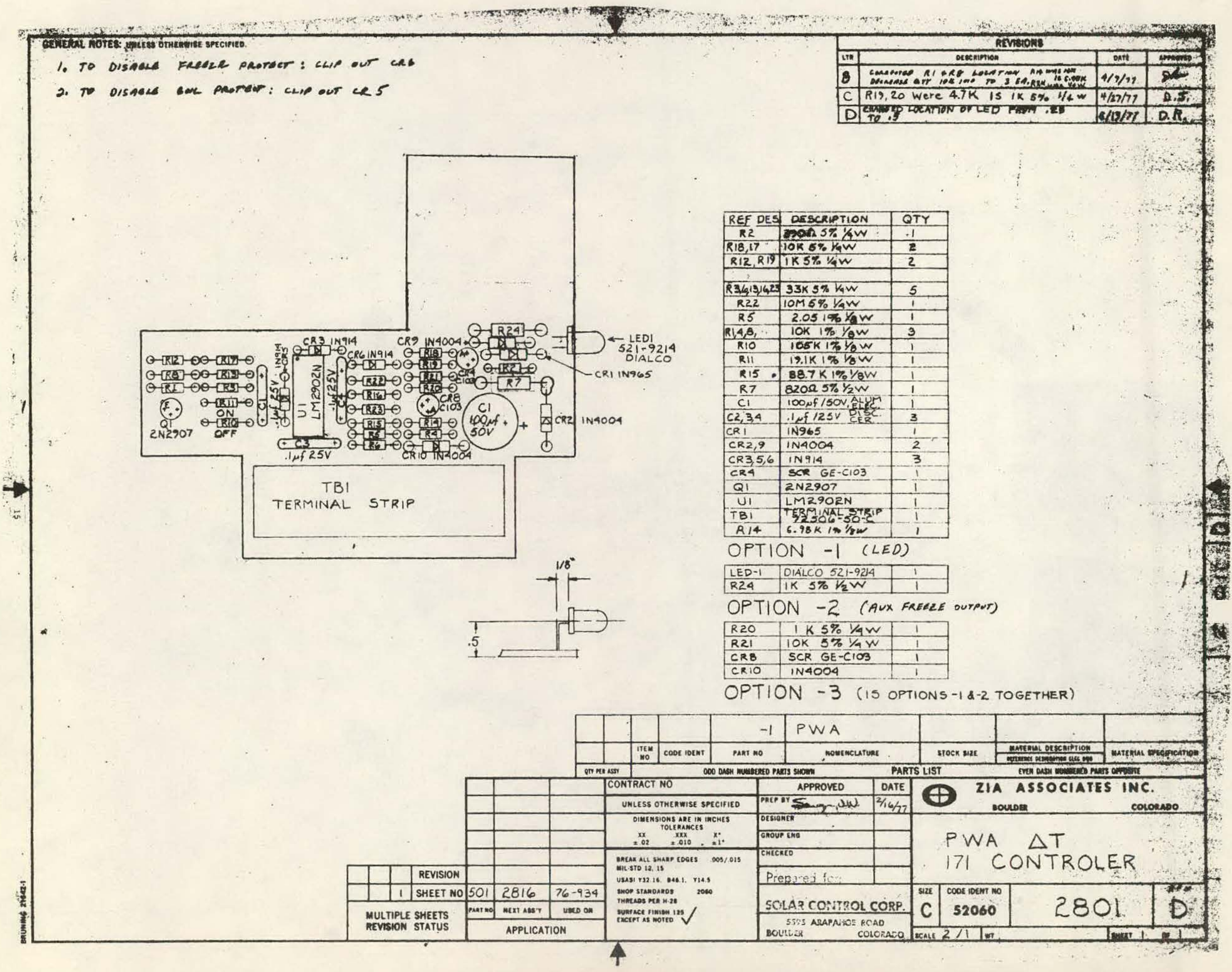




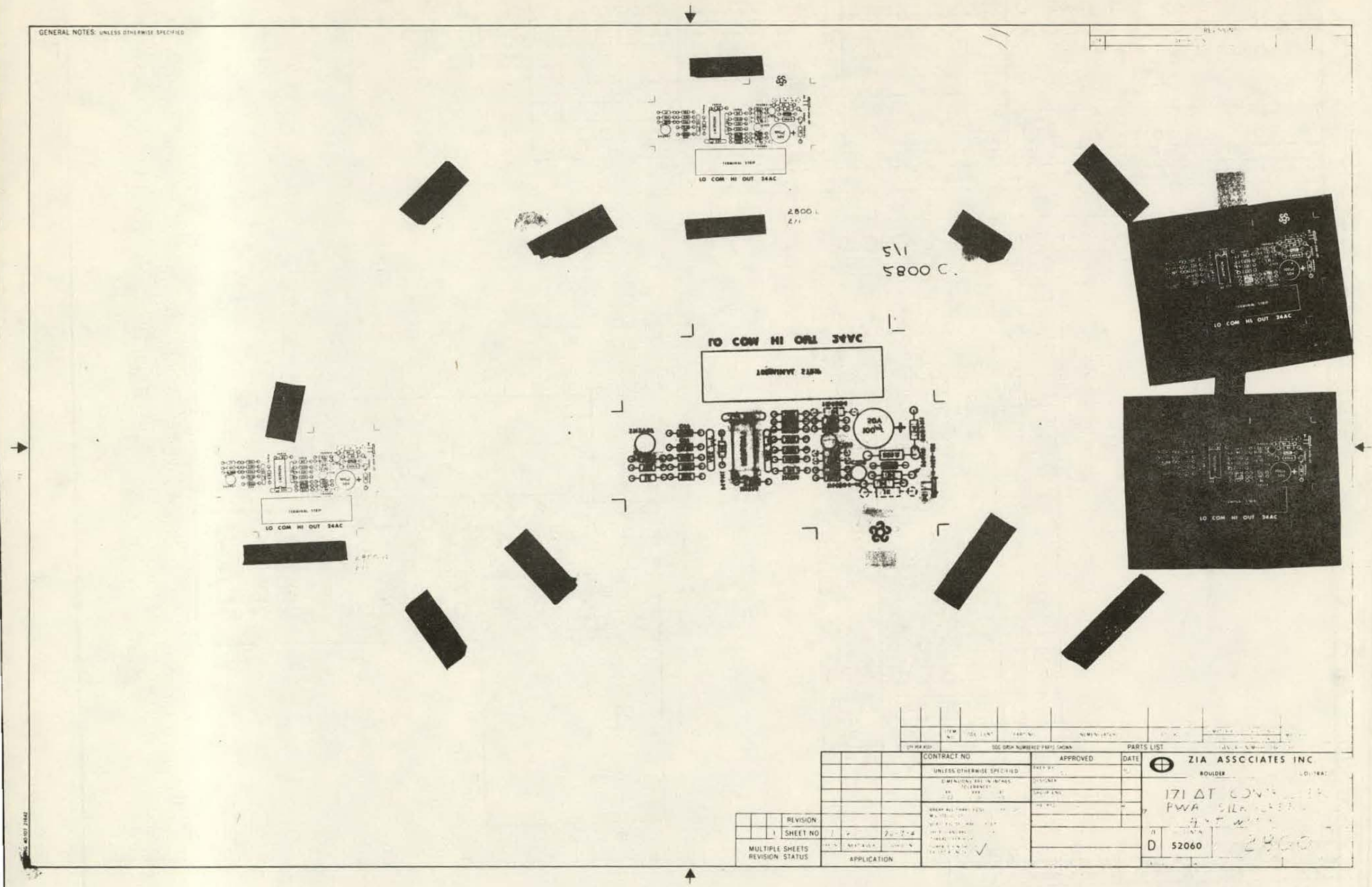




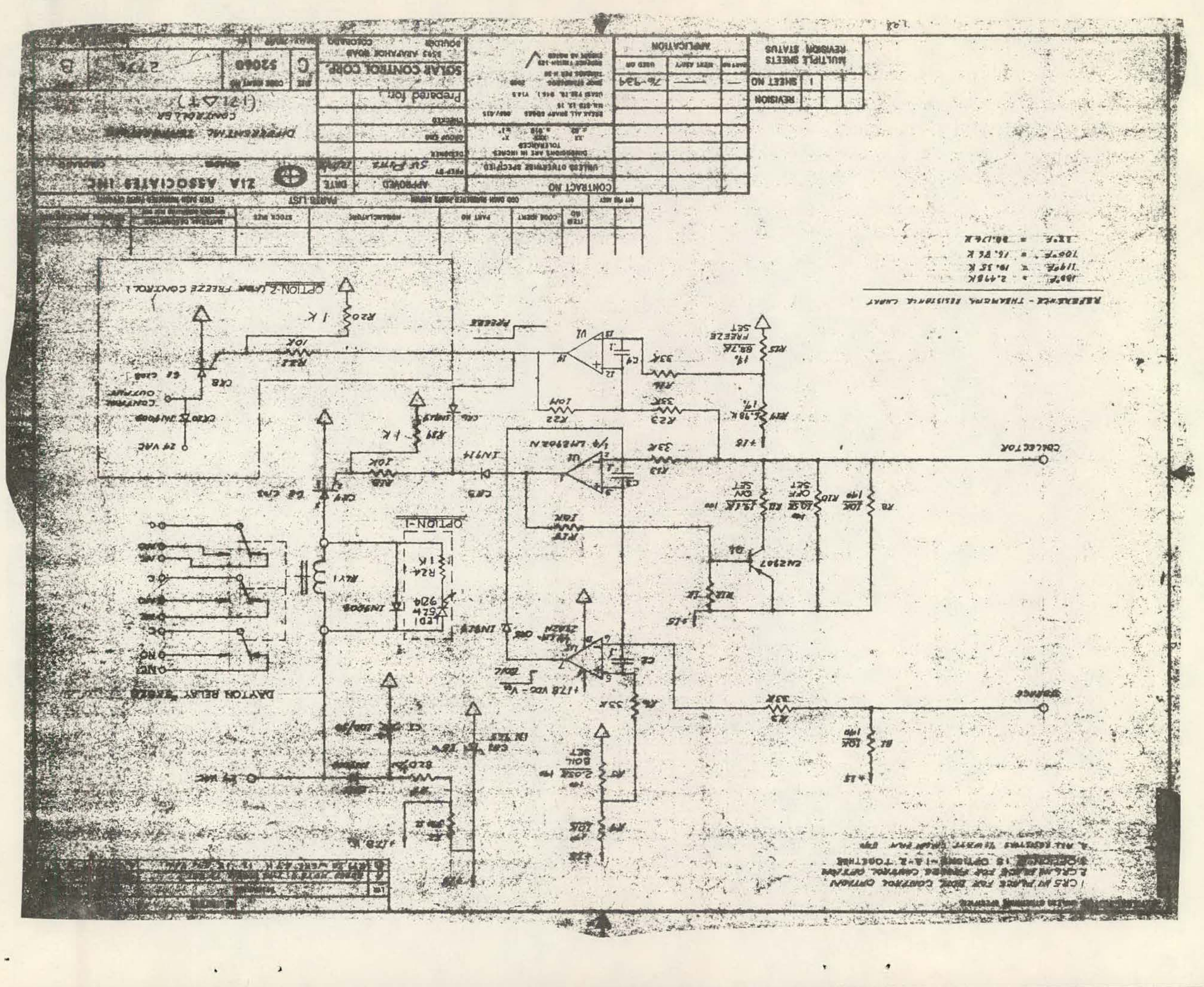




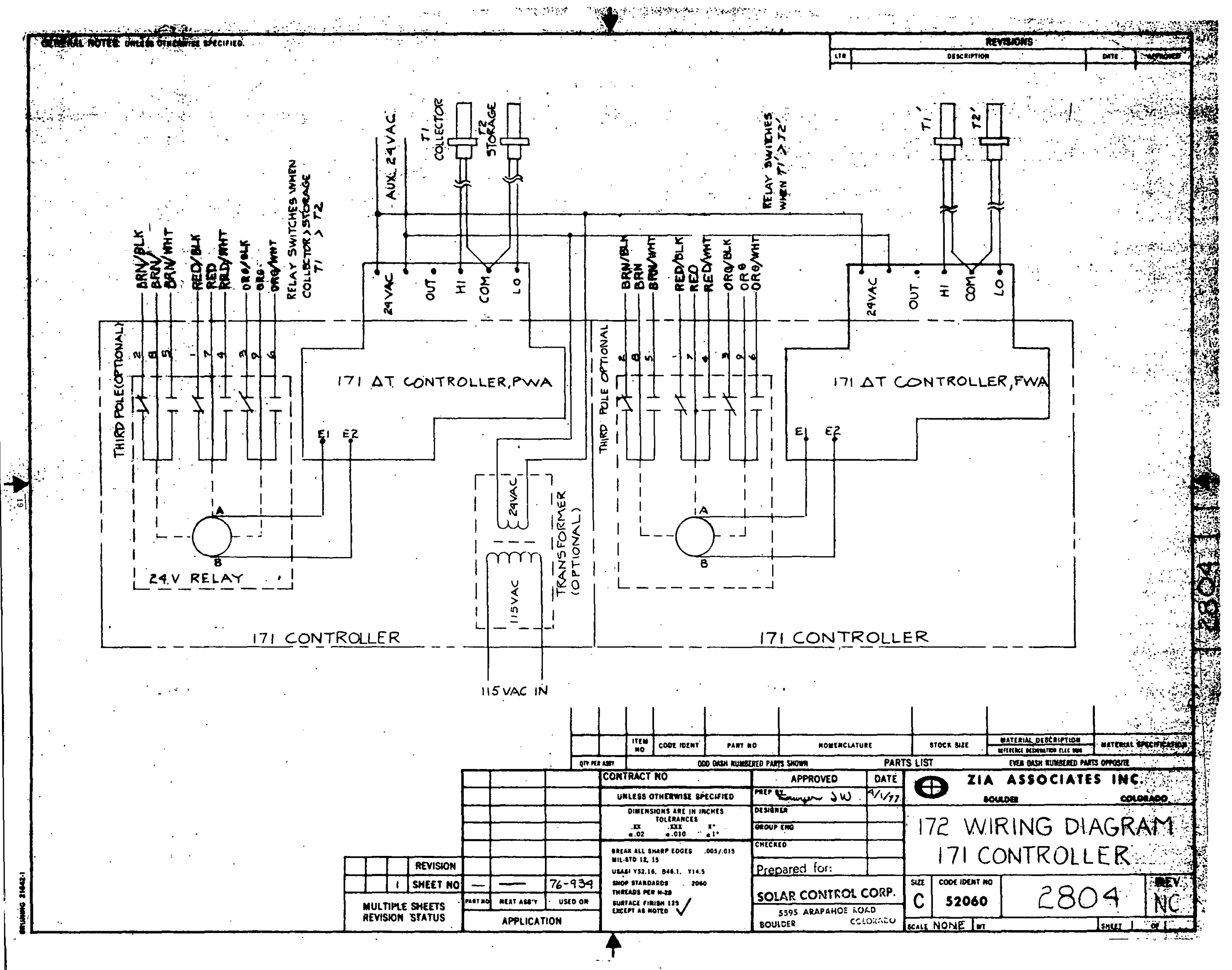




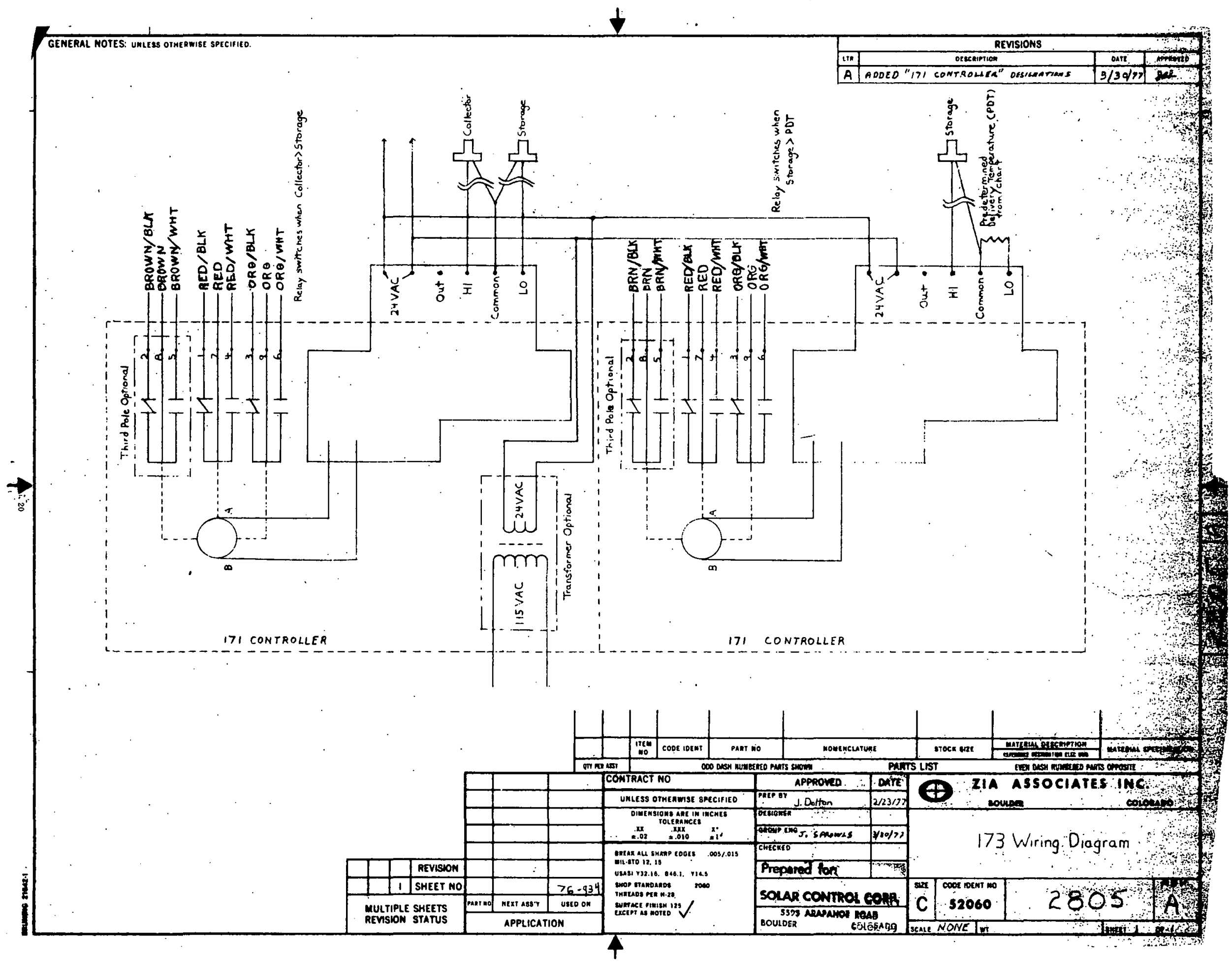




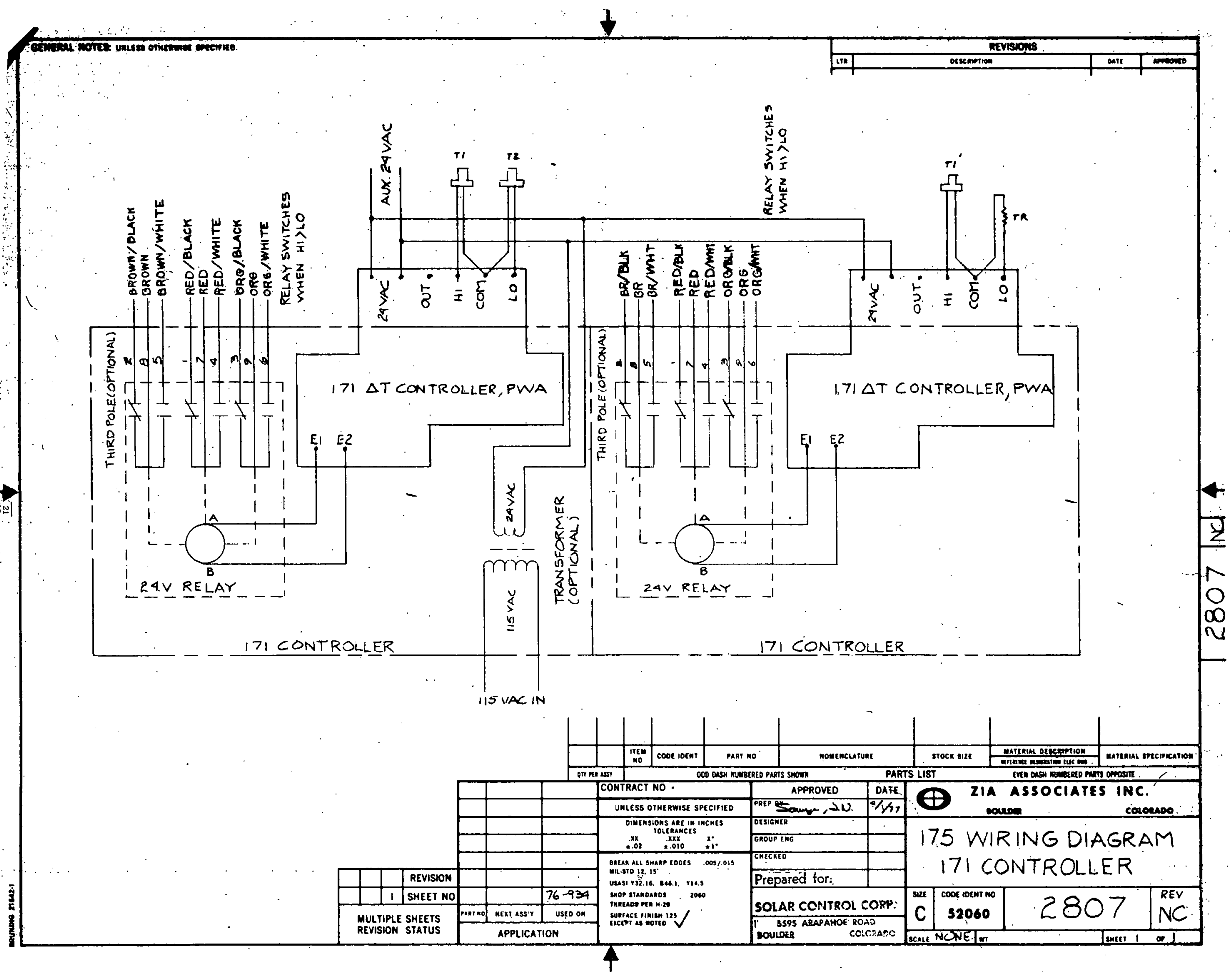




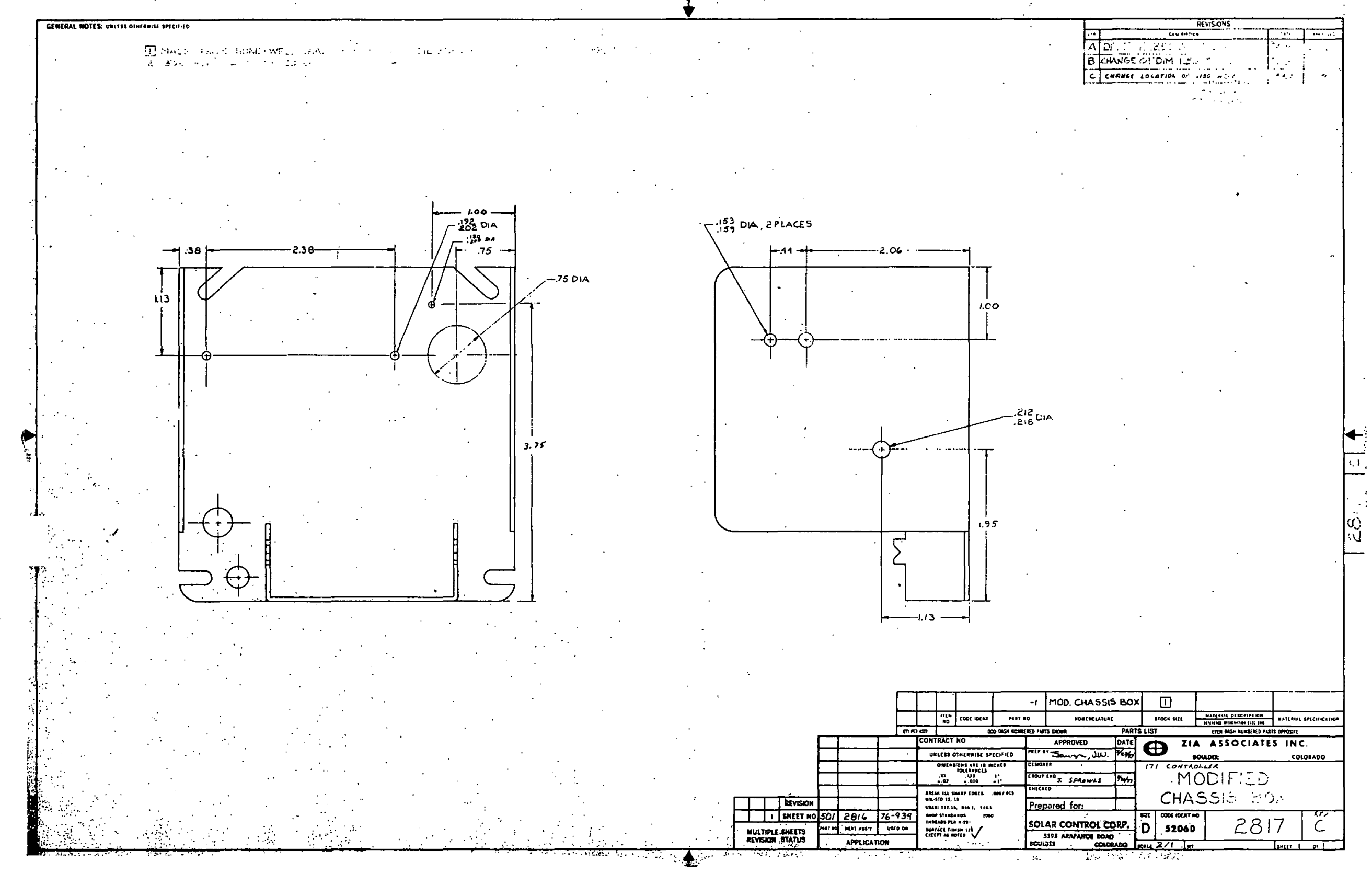




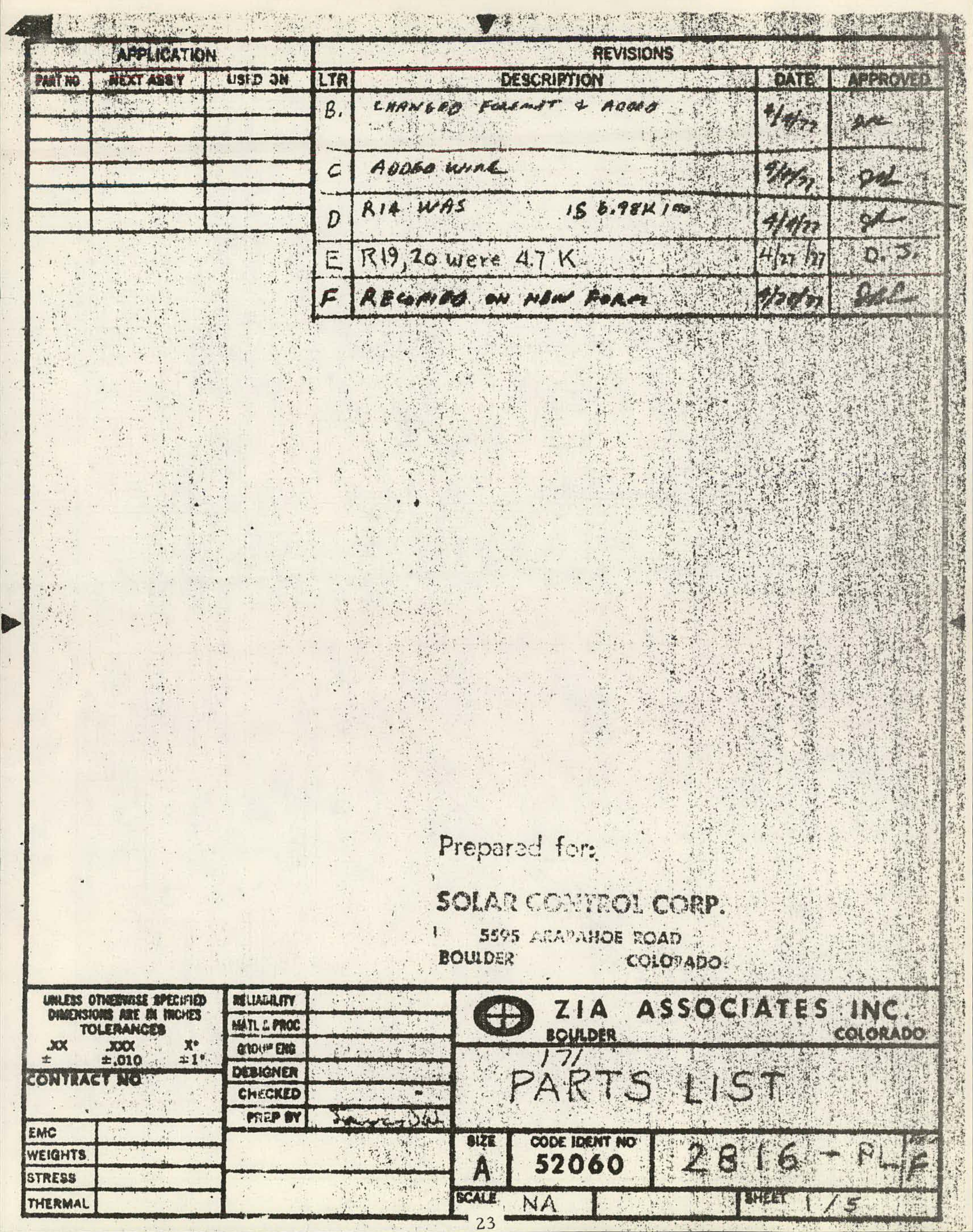




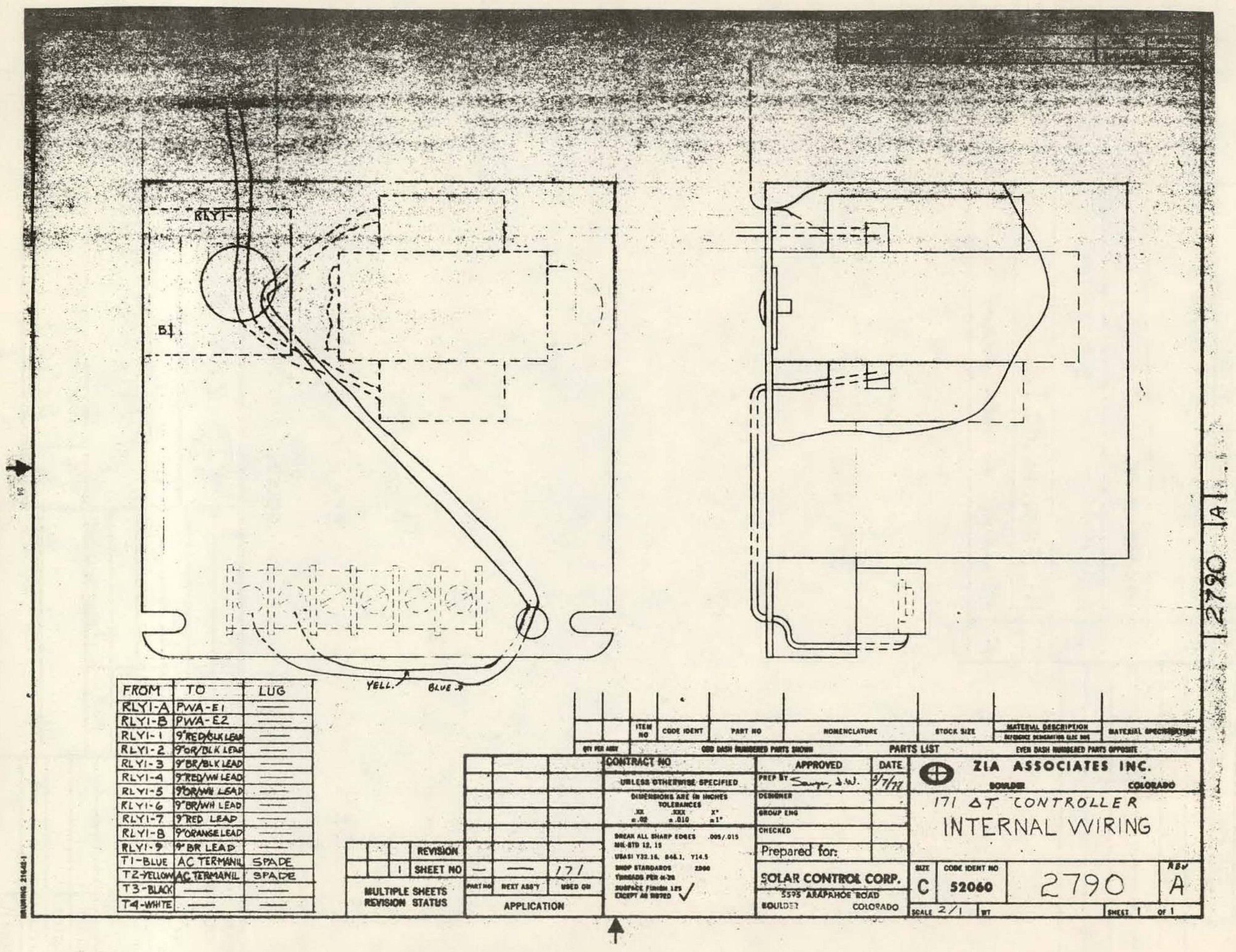




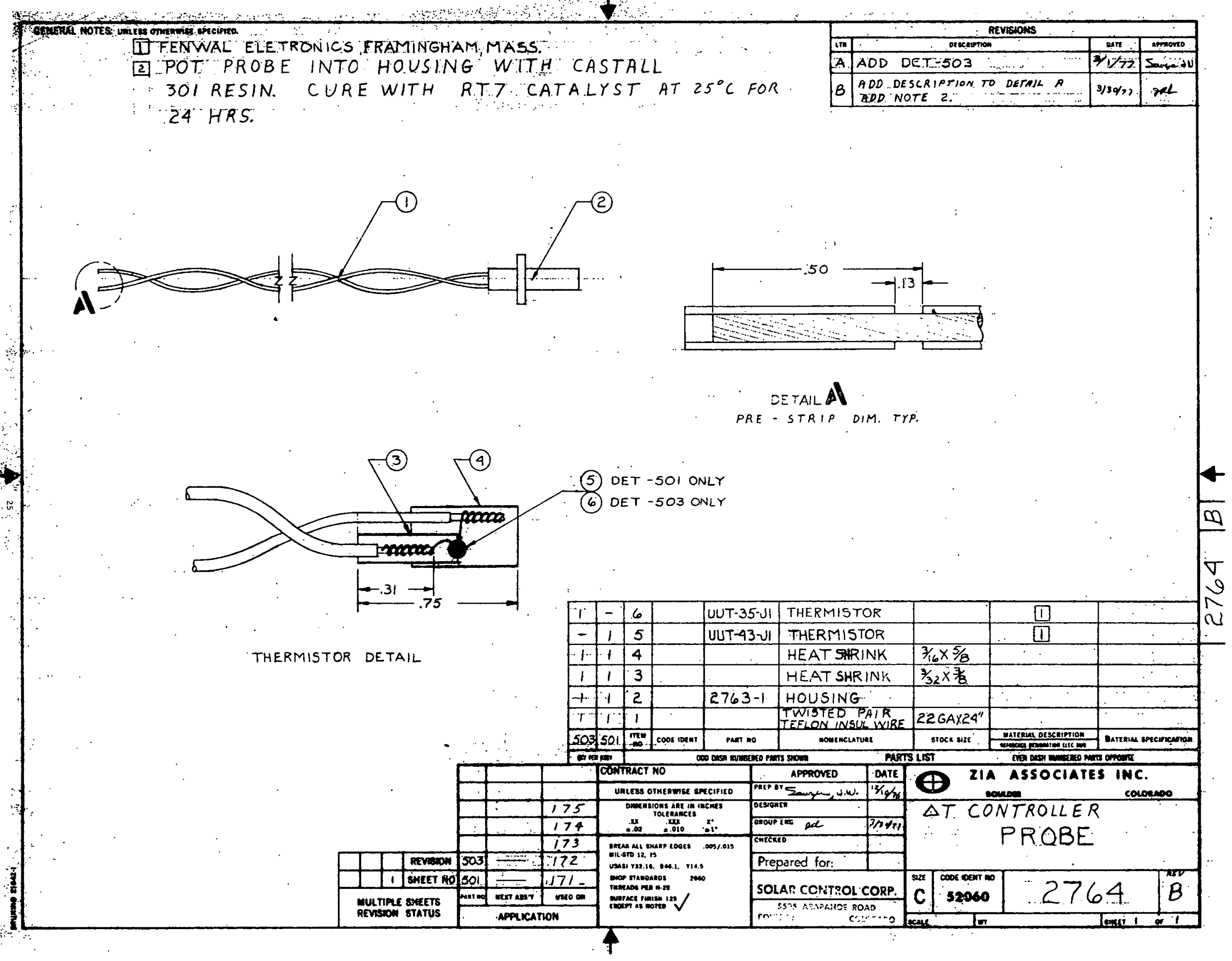




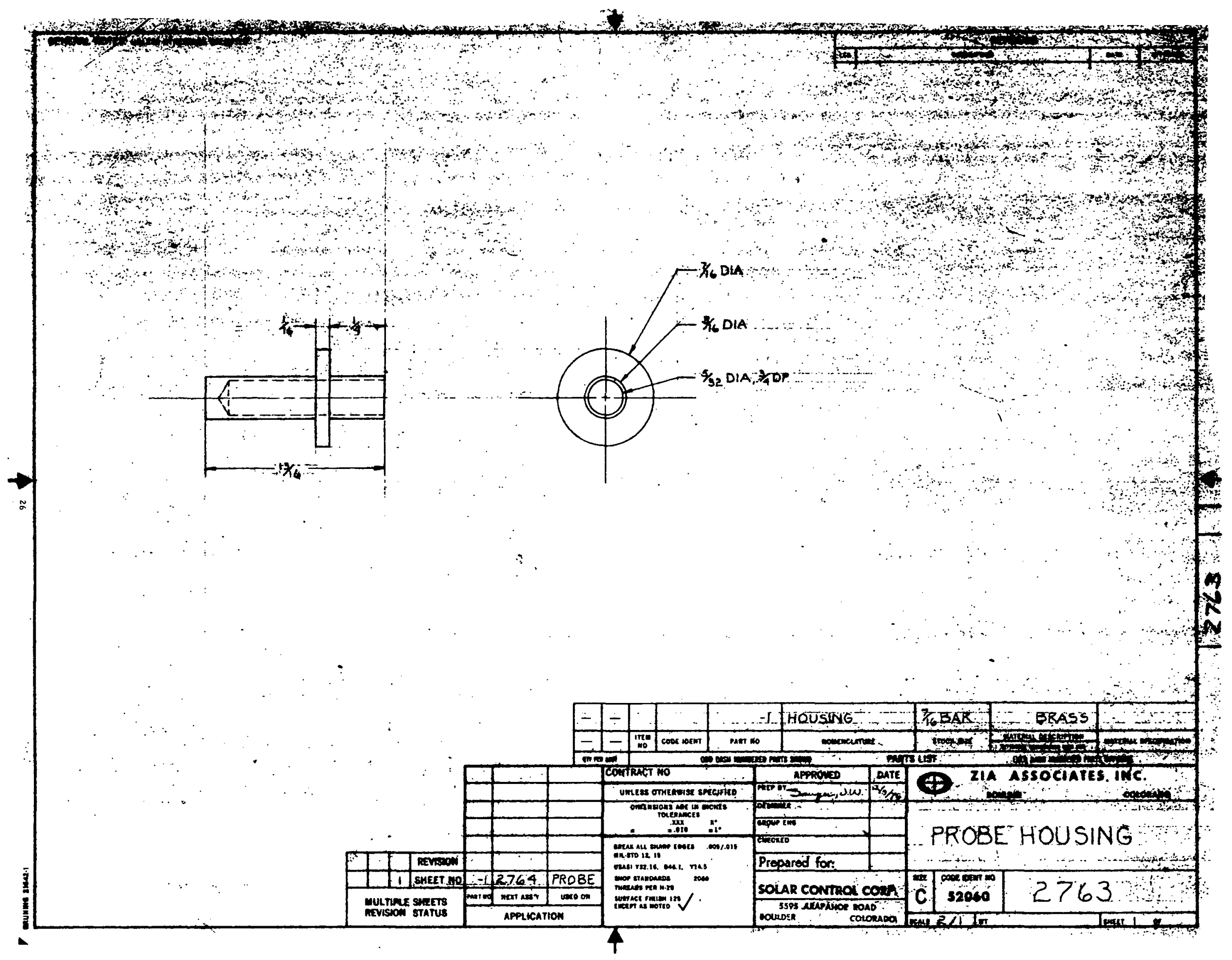




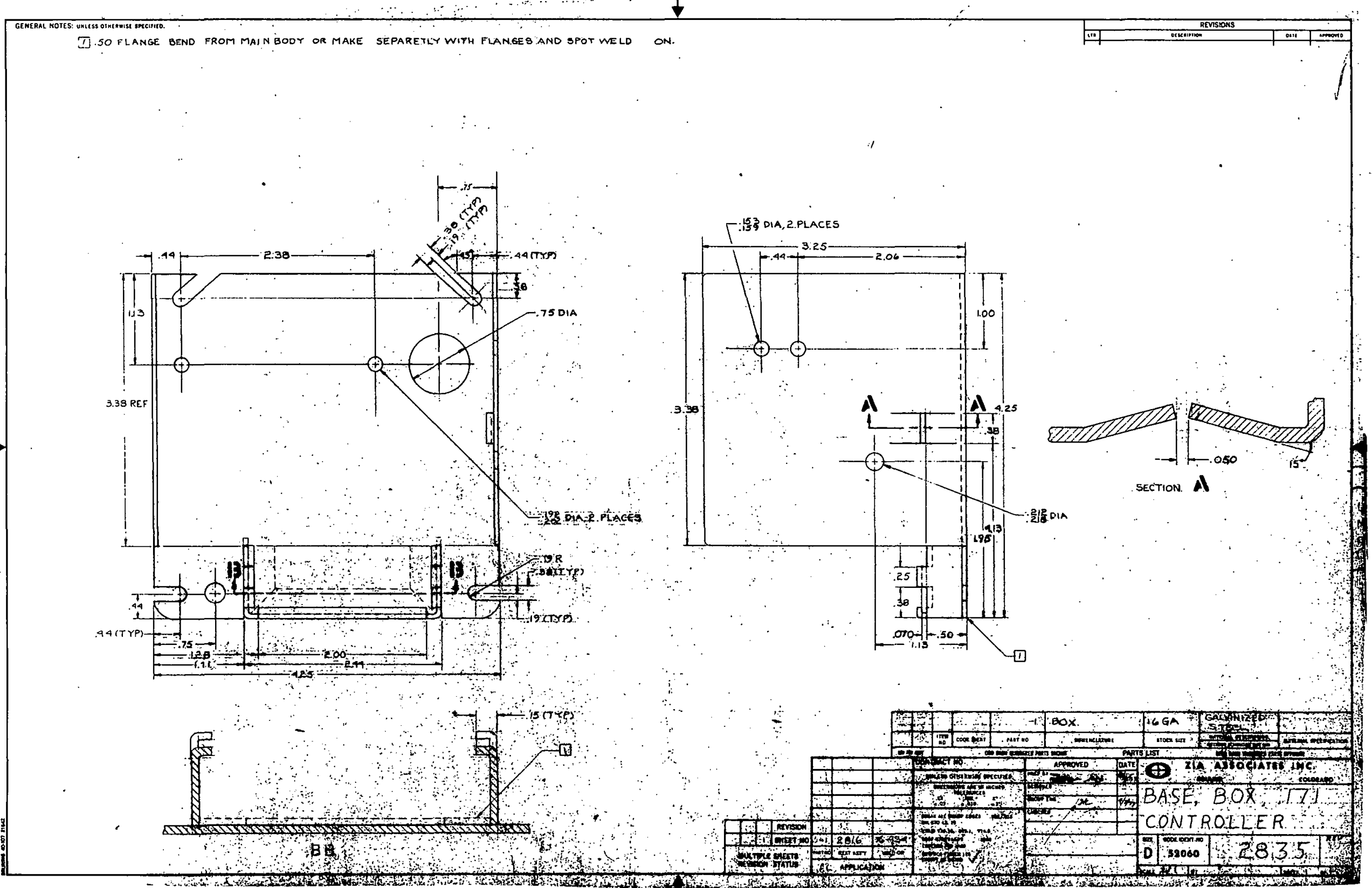




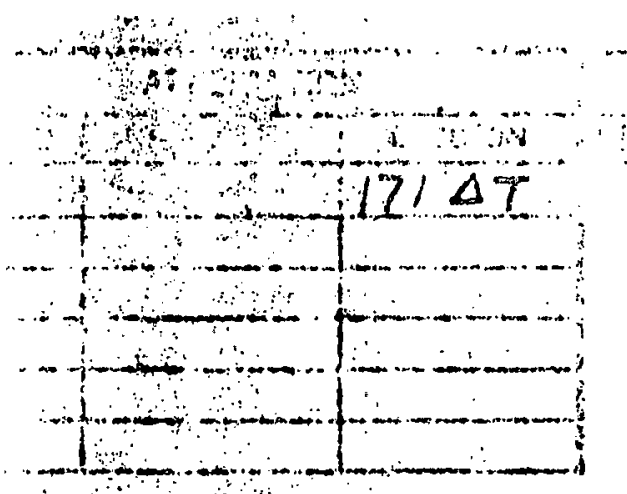

Consts: OF:

1) contrit $54 \mathrm{~s}$

2) JHEET if?

$1 \% 2$

3) $T I T L E \geqslant 96 E$

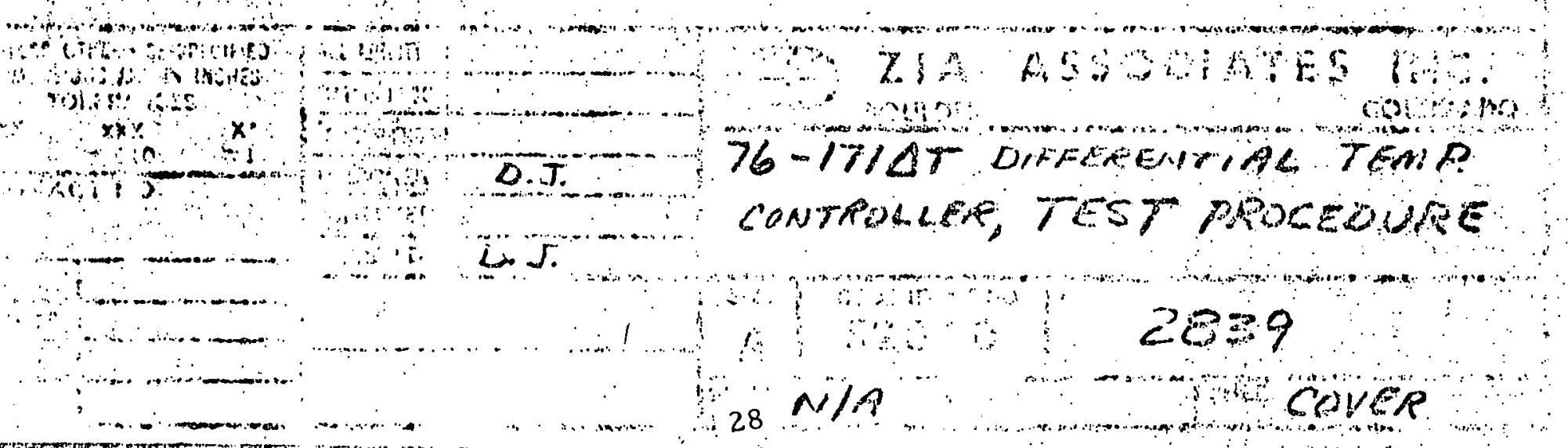




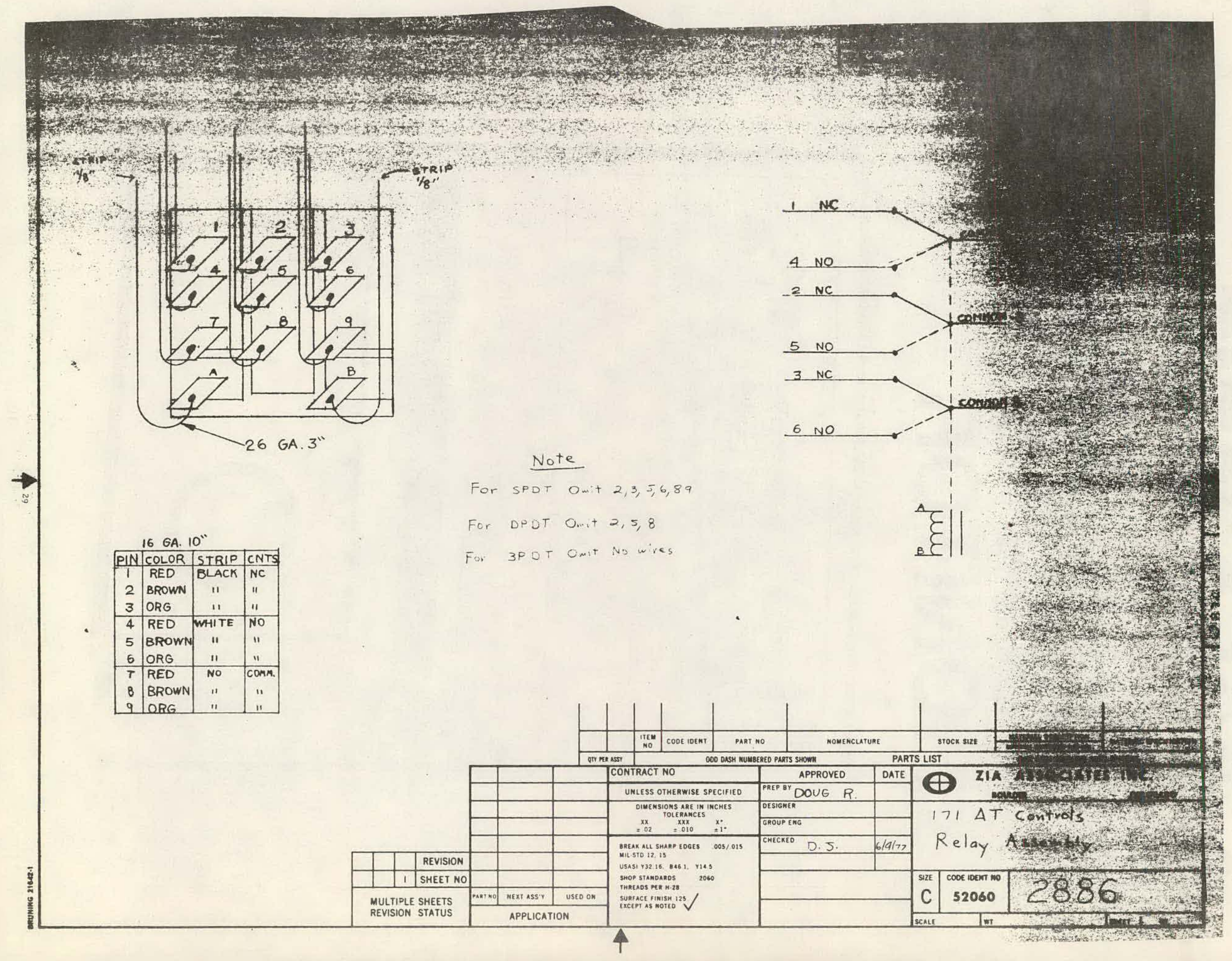



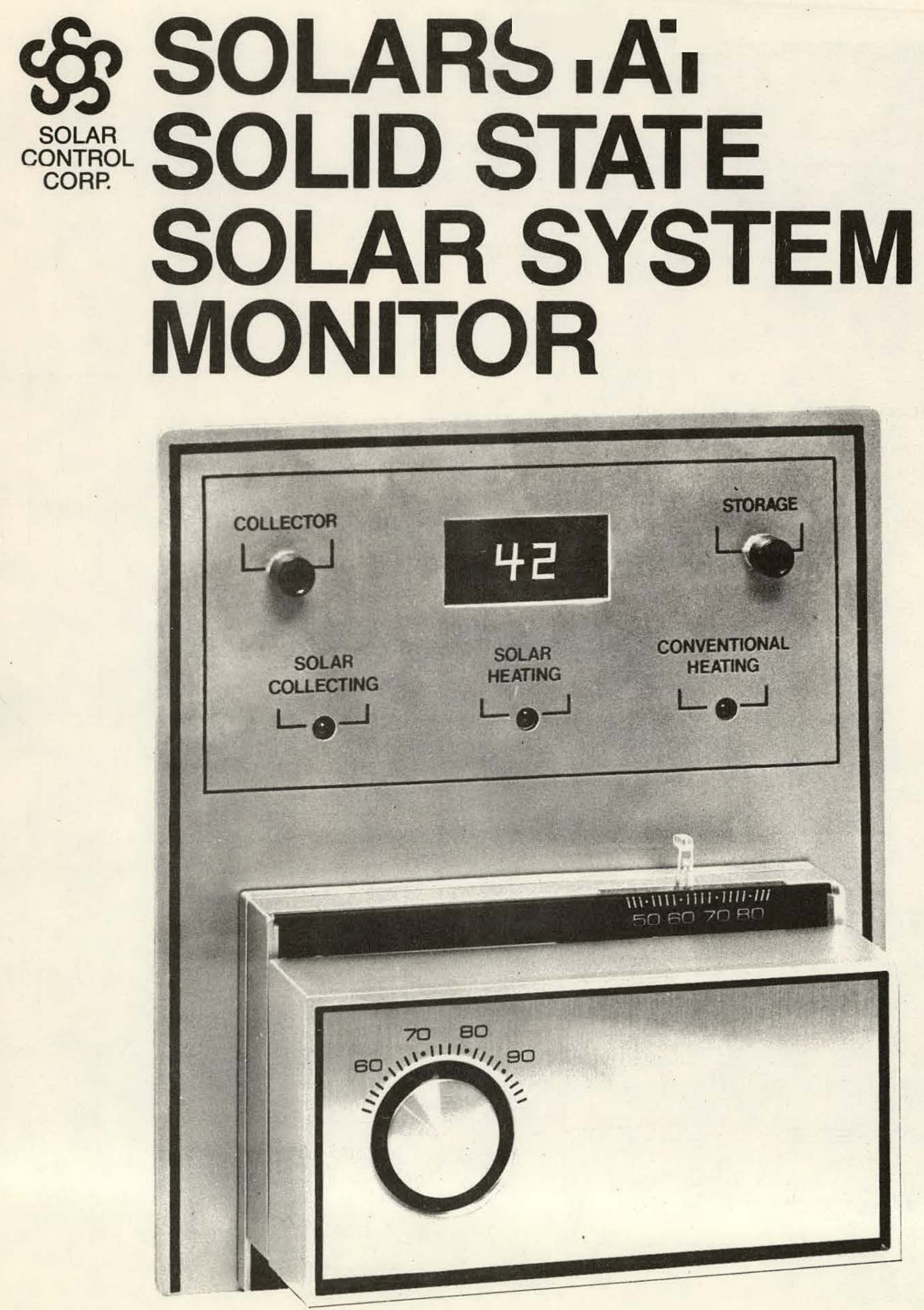


\section{Model 77-180 \\ Solid State Solar System Monitor}

\section{Description}

The model $77-180$ is a solid state temperature and system function monitor that provides the homeowner with accurate outdoor, indoor, collector and storage temperature readings.

The outdoor temperature is continuously indicated by a 0.3 inch liquid crystal display. Collector and storage temperatures may be selected by depressing the appropriate button. The control thermostat provides room temperature as well as two stage signals to the home's solar and back-up heating system.

The solar heating system's current mode of operation is indicated by three light emitting diodes which give a bright red indication of solar collecting, solar distribution, or back-up heating function.

The Solarstat uses less than 10 watts at $24 \mathrm{VAC}$ and is compatible with all conventional HVAC equipment.

\section{Specifications}

Input voltage $\ldots \ldots \ldots \ldots .24$ VAC $\pm 20 \%$.

Input power .......... 10 watts maximum.

Temperature sensors .... Diodes, linear from $-40^{\circ}$ to $+199^{\circ} \mathrm{F}$.

Displays ............. Temperature -0.3 inch liquid crystal, 3 digits plus $(-)$ sign. Mode of operation-3 light emitting diodes.

Display range . . . . . Temperature $--40^{\circ}$ to $+199^{\circ} \mathrm{F}$.

Accuracy $\ldots \ldots \ldots \ldots \ldots \pm 0.5 \%$ one count on least significant digit.

Measurement points ..... Outdoor, collector, storage and room.

Thermostat........... Two stage heating. Optional: two stage heating, one stage cooling and fan on/off.

Solarstat operating

temperature ........... $32^{\circ}$ to $120^{\circ} \mathrm{F}$.

Size ................. $8^{\prime \prime} \times 71^{\prime \prime} \times 2 \frac{1}{1 \prime \prime}$.

Weight $\ldots \ldots \ldots \ldots \ldots \ldots 2$ lbs.

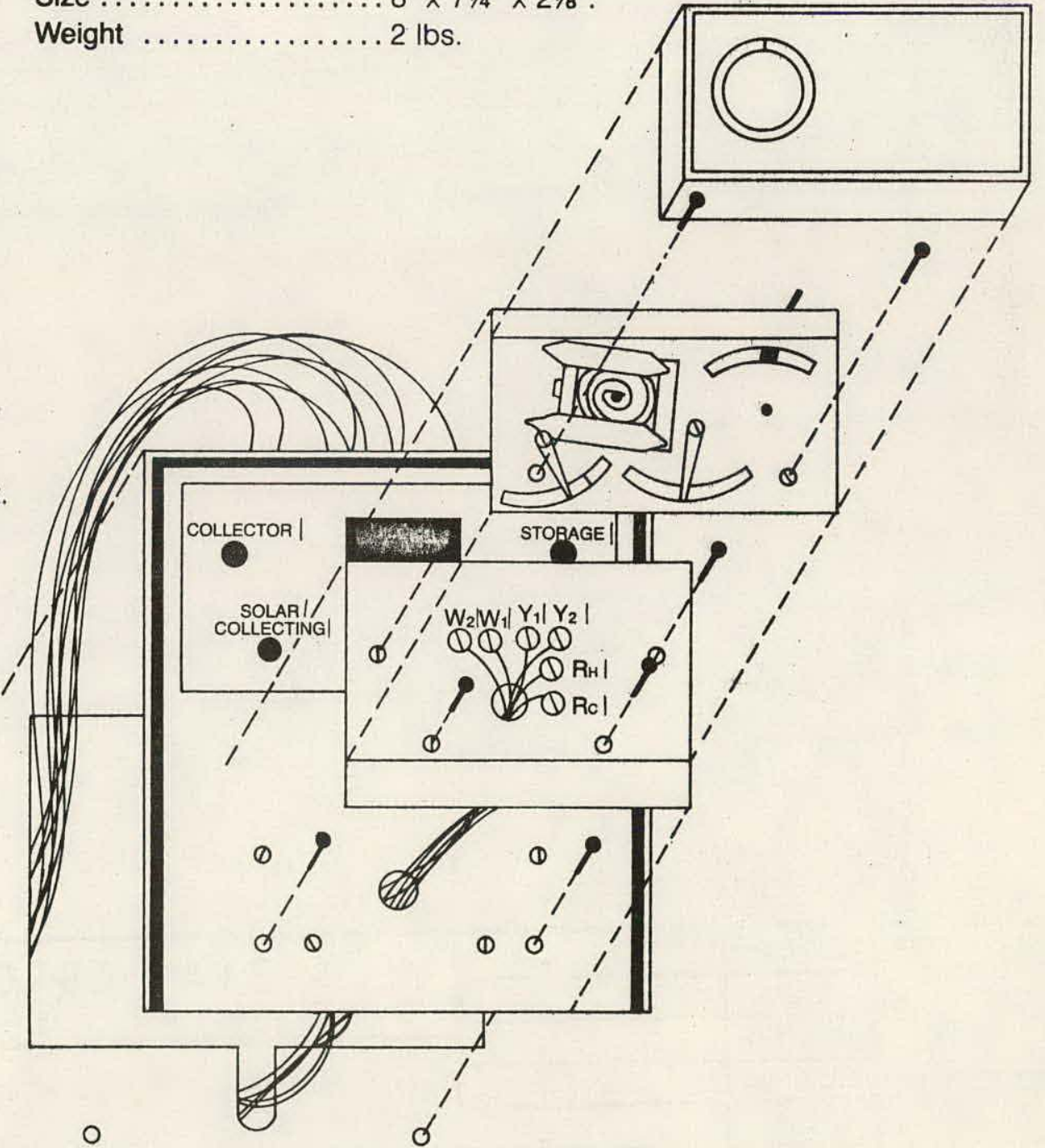




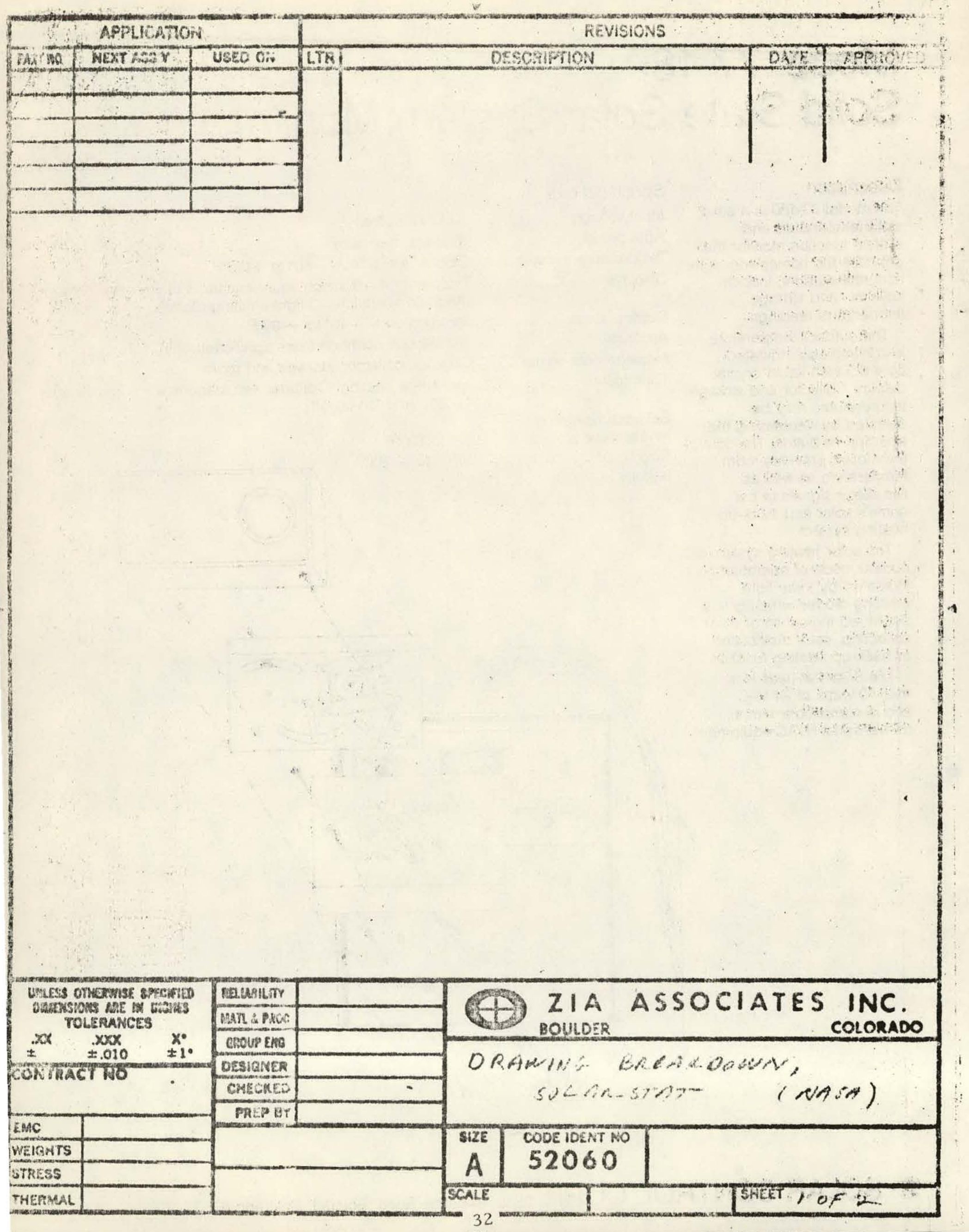


PRERREO OO D AUGUSTO _. DRAWING BREAKDOIYN Gare $1 / 2177$

prarct Solars lat (NASA)

wiaka $26-93$

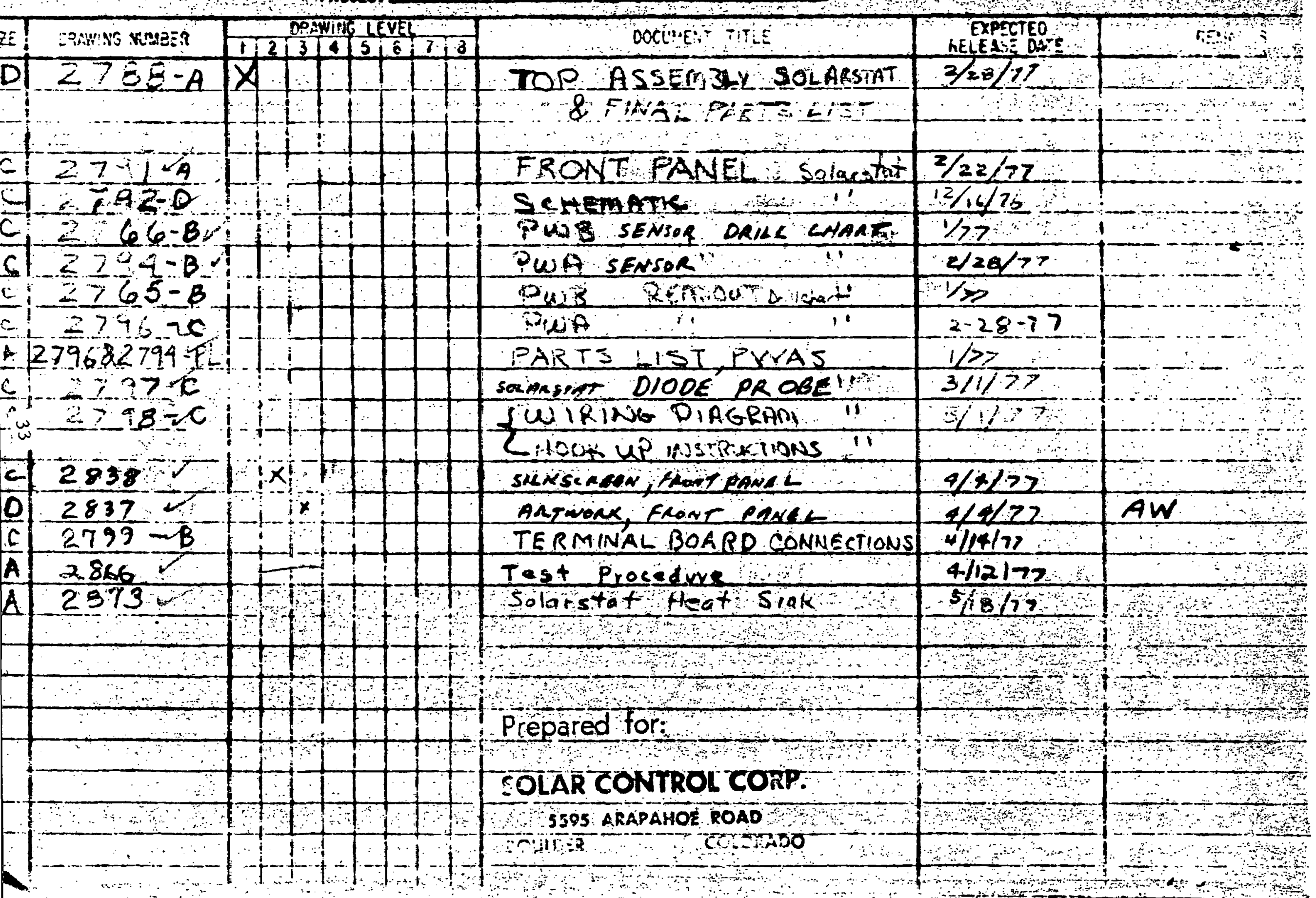


FIELD INSTALLATION MAGRAMA

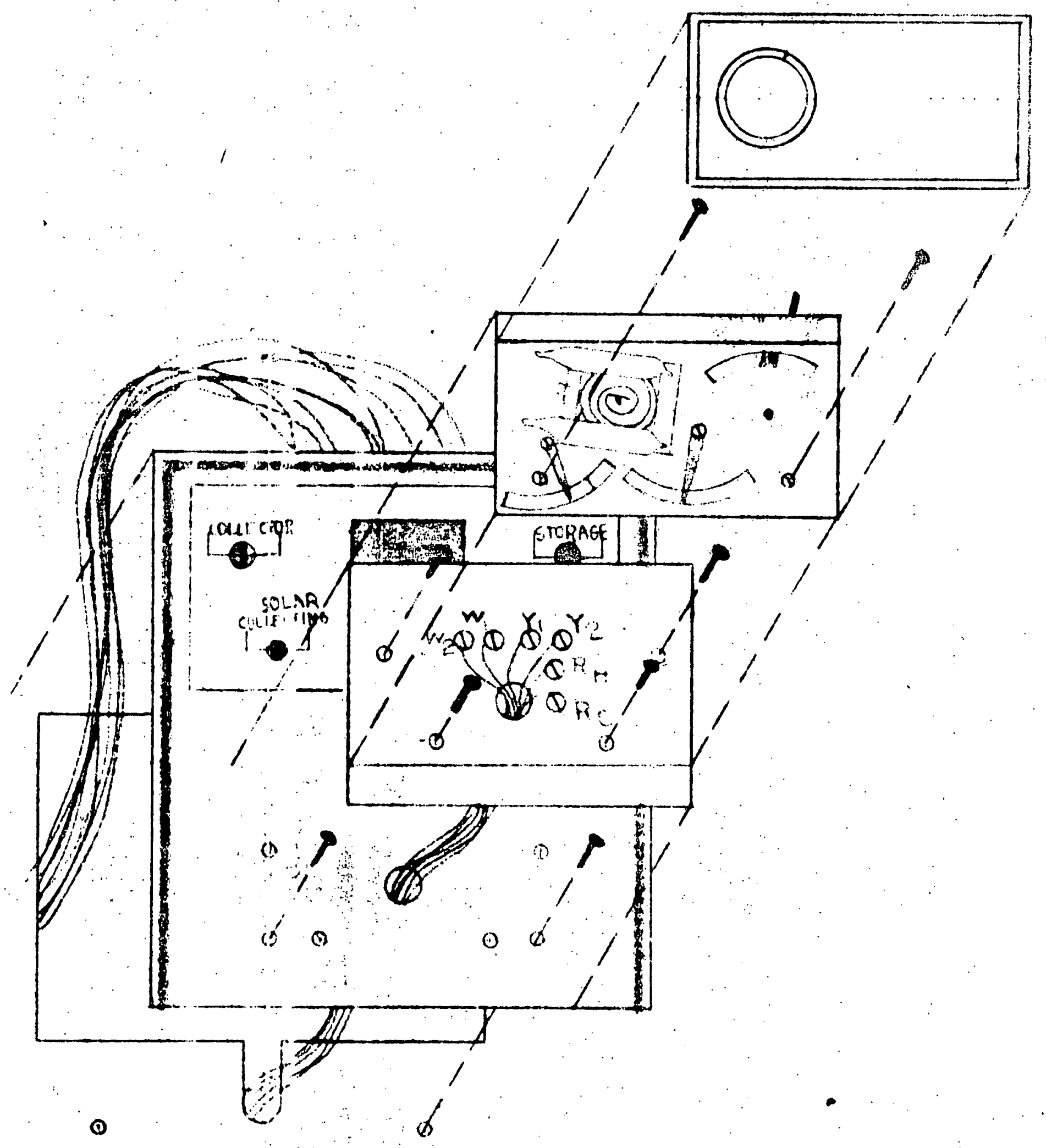




\begin{tabular}{|c|c|}
\hline "J". & INTERFACE \\
\hline$J 1$ & $24 \vee A C$ \\
\hline$\sqrt{2}$ & $24 \vee A C$ \\
\hline 13 & GROUND \\
\hline$\sqrt{4}$ & STORAGE PROBE \\
\hline$\lrcorner 5$ & STORAGE PROBE NEG. \\
\hline$J 6$ & COLLECTOR PROBE \\
\hline$J T$ & COLLECTOR PROBE NEG. \\
\hline 」 8 & NORMAL PROBE \\
\hline J 9 & NOFMAL PKOBE NEG. \\
\hline$j 10$ & $\begin{array}{l}\text { SOLAR COLLECTING RELAY - } \\
24 \vee A C \text { OR POS DC }\end{array}$ \\
\hline UII & $\begin{array}{l}\text { SOL AR COLLECTING REIAY- } \\
24 \vee A C \text { OR NEG DC }\end{array}$ \\
\hline$J 12$ & $\begin{array}{l}\text { IR HEATING RELAY } \\
\text { AC OR POS. DC }\end{array}$ \\
\hline 113 & $\begin{array}{l}\text { SOLAR HEATING RELAY } \\
24 \text { VAC OR NEG PC. }\end{array}$ \\
\hline$J 14$ & $\begin{array}{l}\text { CONVENTIONAL HEAT ING } \\
\text { RELAY-24VACORPOS. OC }\end{array}$ \\
\hline$\sqrt{15}$ & $\begin{array}{l}\text { CONVENTIONAL HEATING } \\
\text { RELAY-2AVAC OR NEG. DC }\end{array}$ \\
\hline
\end{tabular}

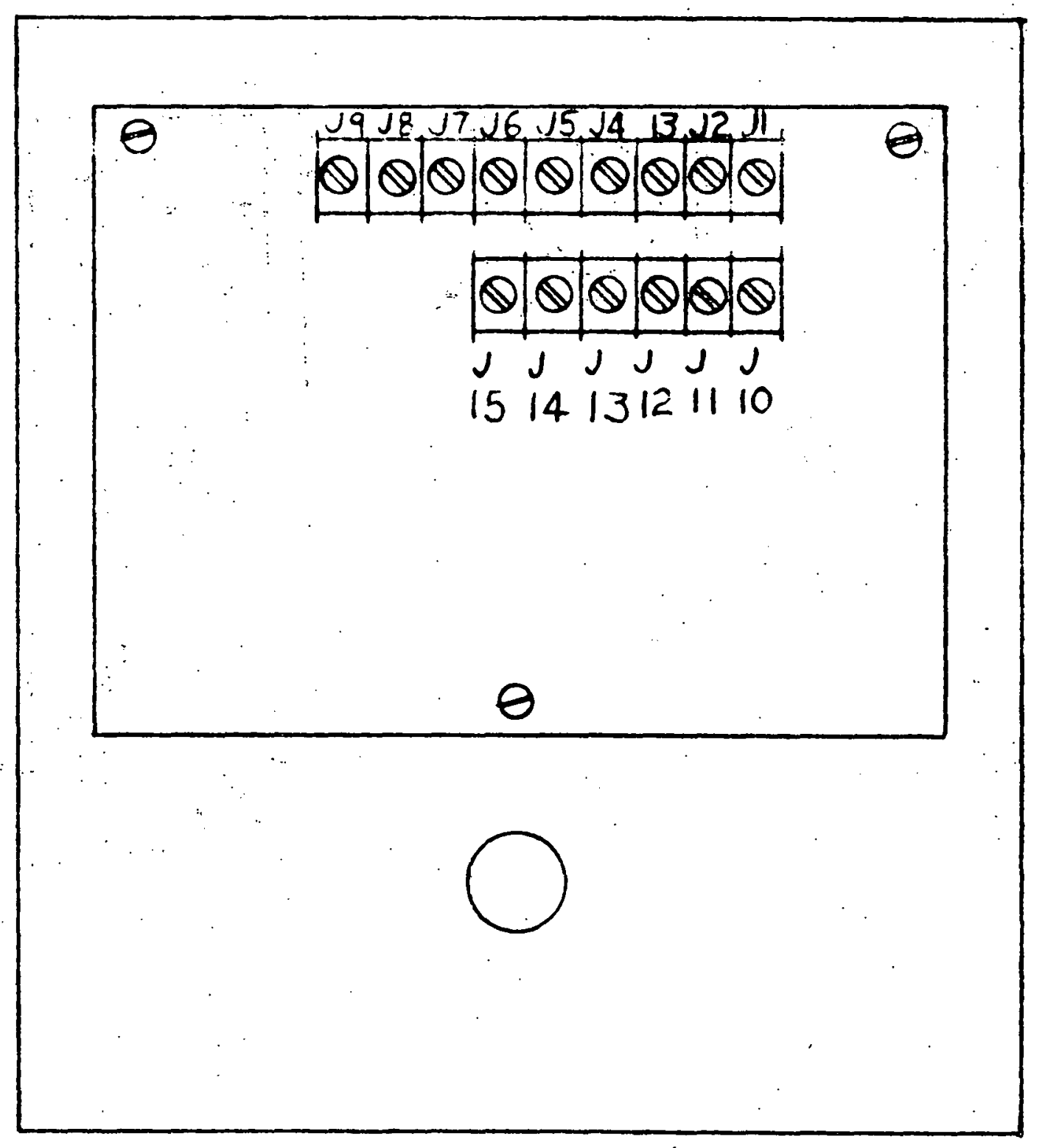




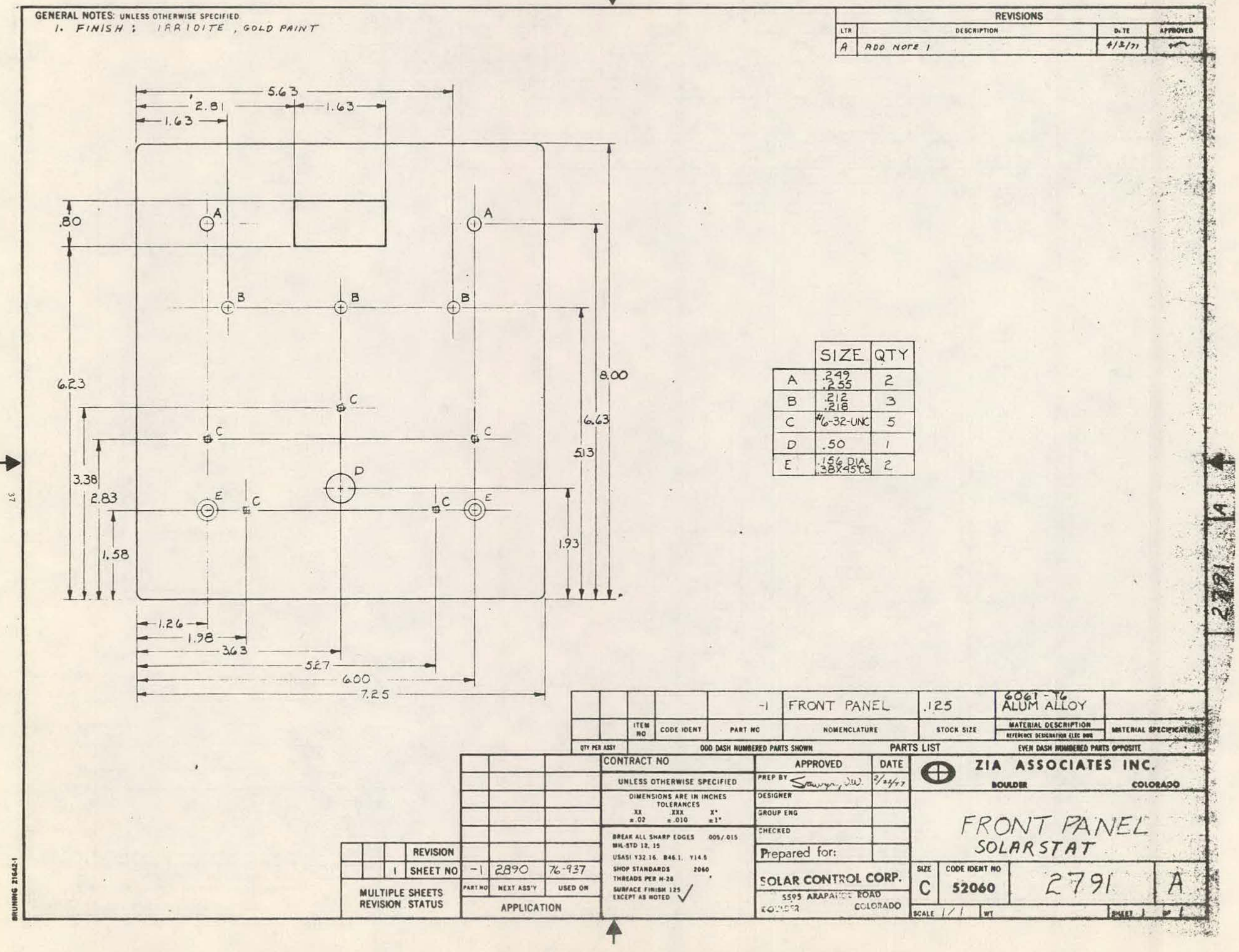



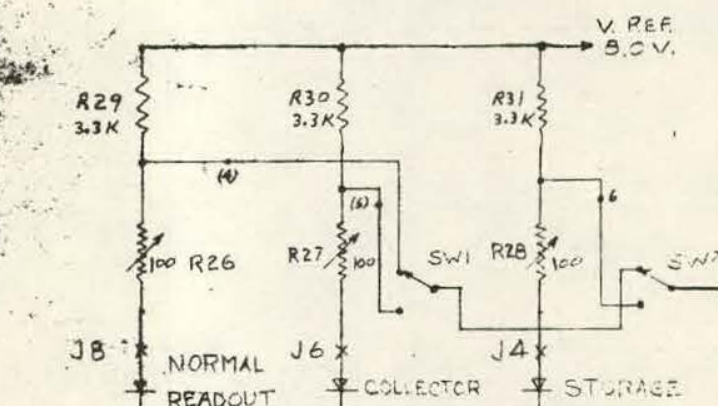

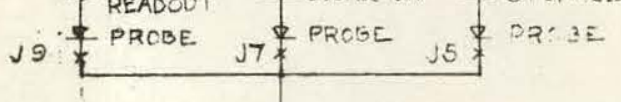

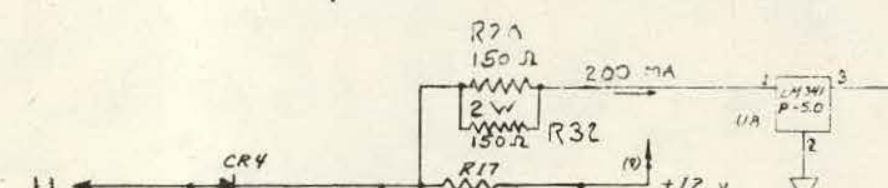

870
$150 \Omega$

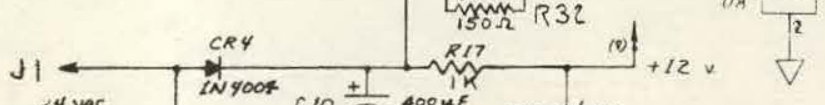

$4 \mathrm{WAC}\left[\begin{array}{l}\text { LNYOOR C10 } \\ \text { CRS }\end{array}\right.$

$\frac{120}{\text { INYOot }}$

$\sqrt{3} \longrightarrow]^{(i)(20)}$

C9 + T.5OUF CR7

$\checkmark$

$\left.1 \underbrace{R 16}_{1 \mathrm{~K}} \cdot \frac{2 N 759 A}{(7)}\right|^{-12 \mathrm{v}}$
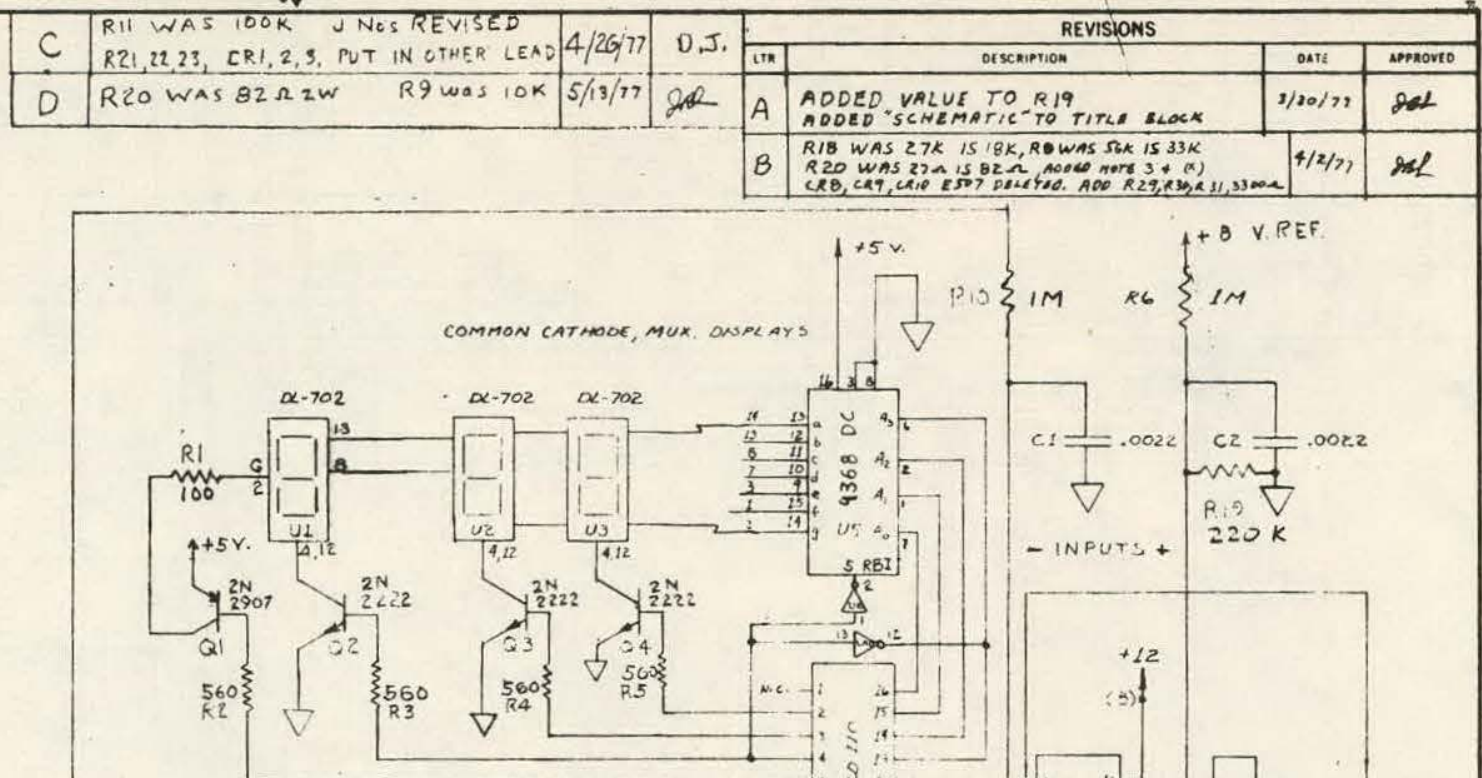

$\left[\begin{array}{ll}+12 \\ +3)+1\end{array}\right.$

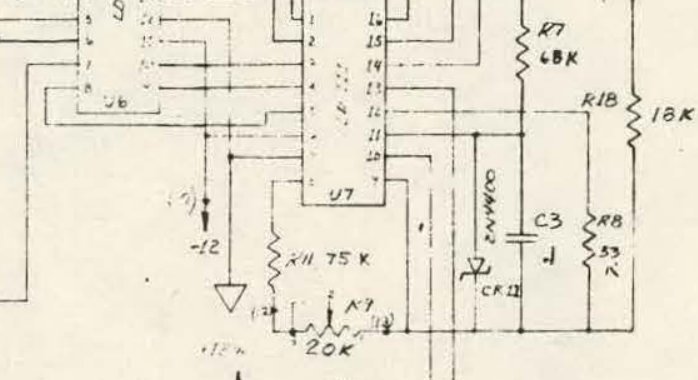

RI2 $33 x^{2}{ }^{8}{ }^{4} e^{4}$

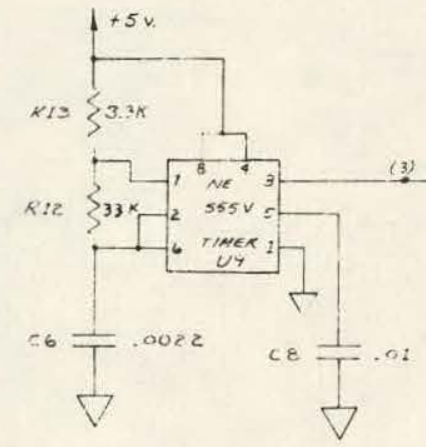

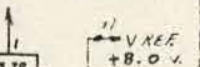

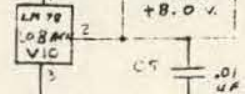
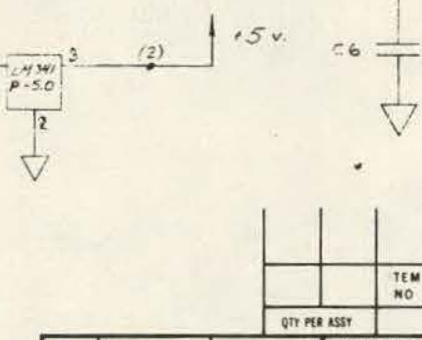

\begin{tabular}{l|l|} 
TEM & CODE IDENT \\
\hline
\end{tabular}

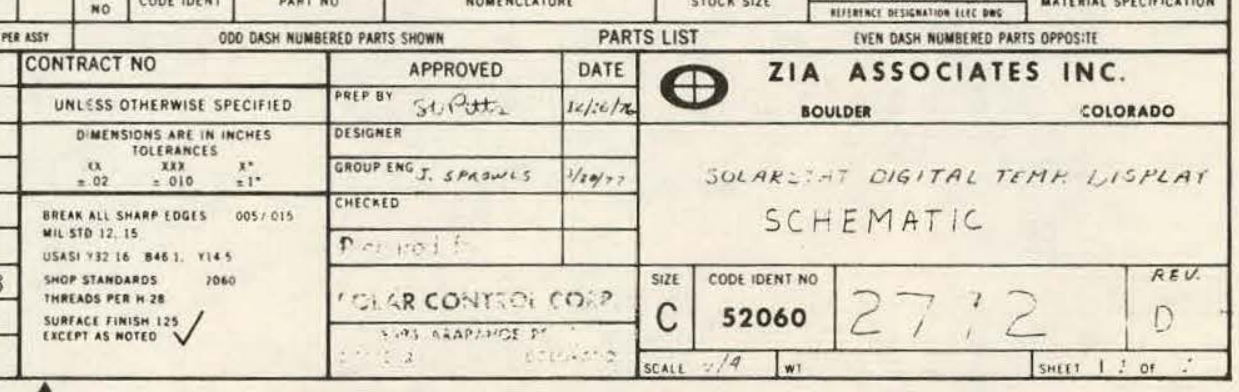

\begin{tabular}{|c|c|c|c|c|c|}
\hline (n) & 2/त्ता & REVISION & & & \\
\hline & 1 & \begin{tabular}{l|l} 
SHEET NO
\end{tabular} & & & 2788 \\
\hline \multirow{2}{*}{\multicolumn{3}{|c|}{$\begin{array}{l}\text { MULTIPLE SHEETS } \\
\text { REVISION STATUS }\end{array}$}} & part no & NEXT ASST & usto on \\
\hline & & & \multicolumn{3}{|c|}{ APPUCATION } \\
\hline
\end{tabular}




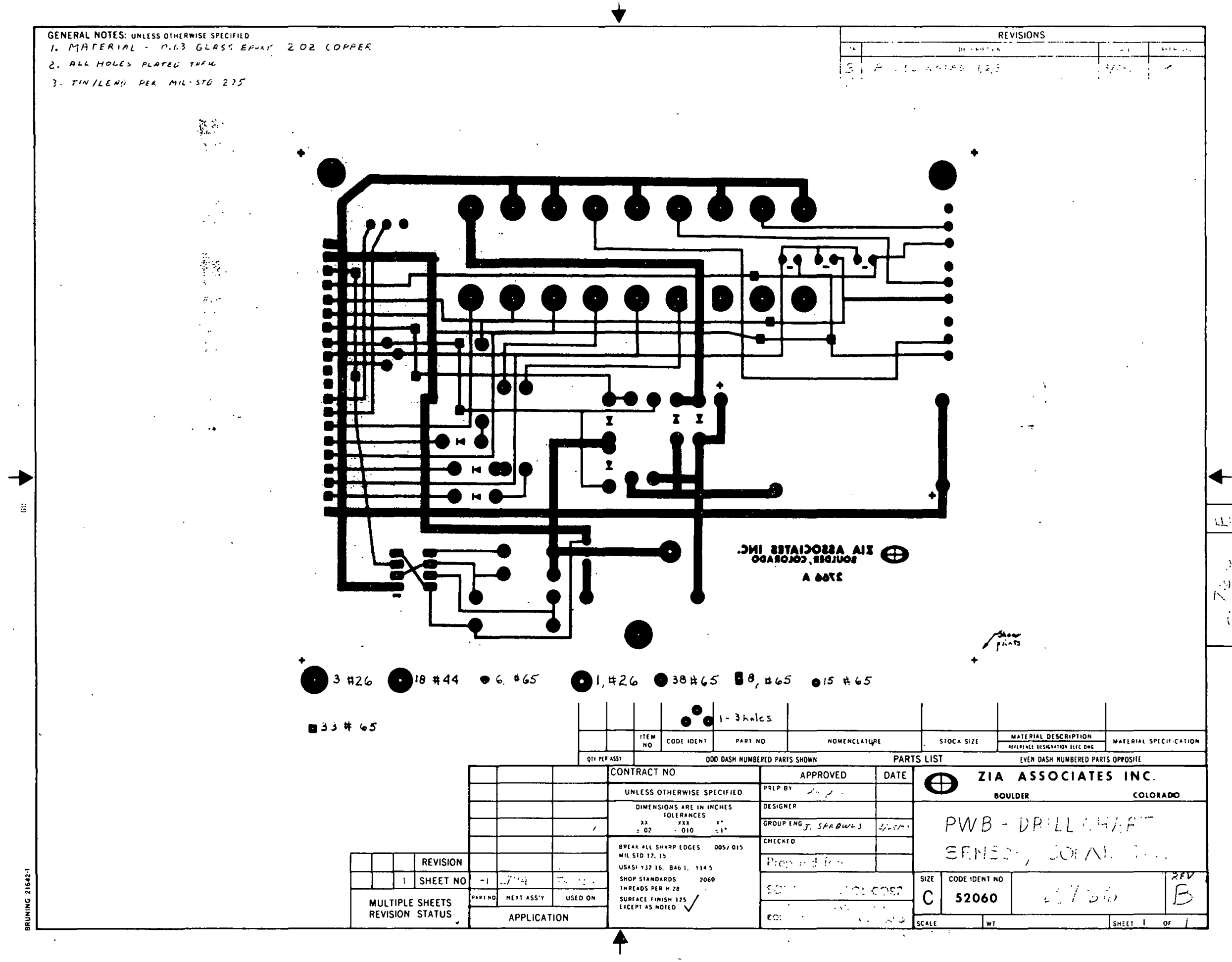




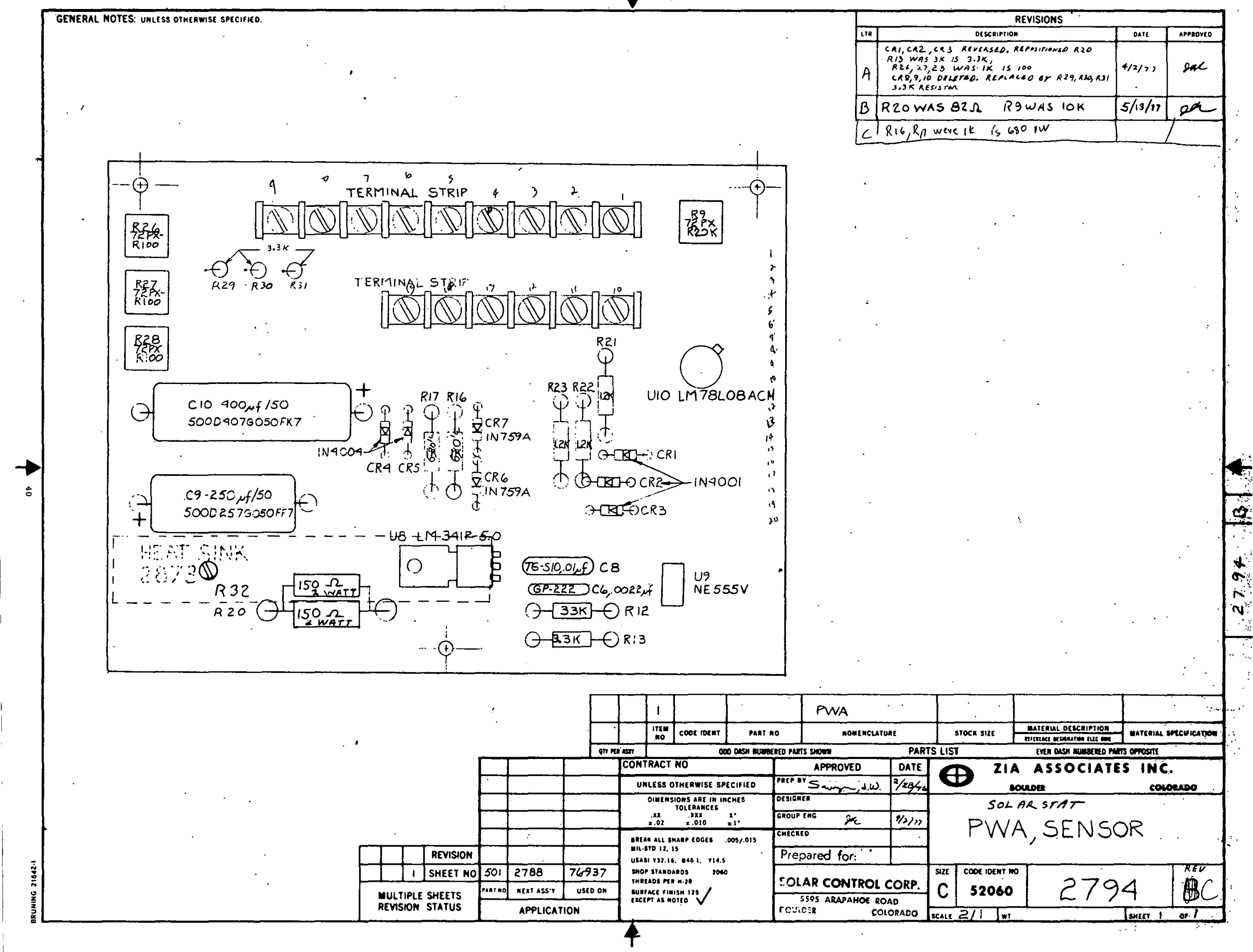




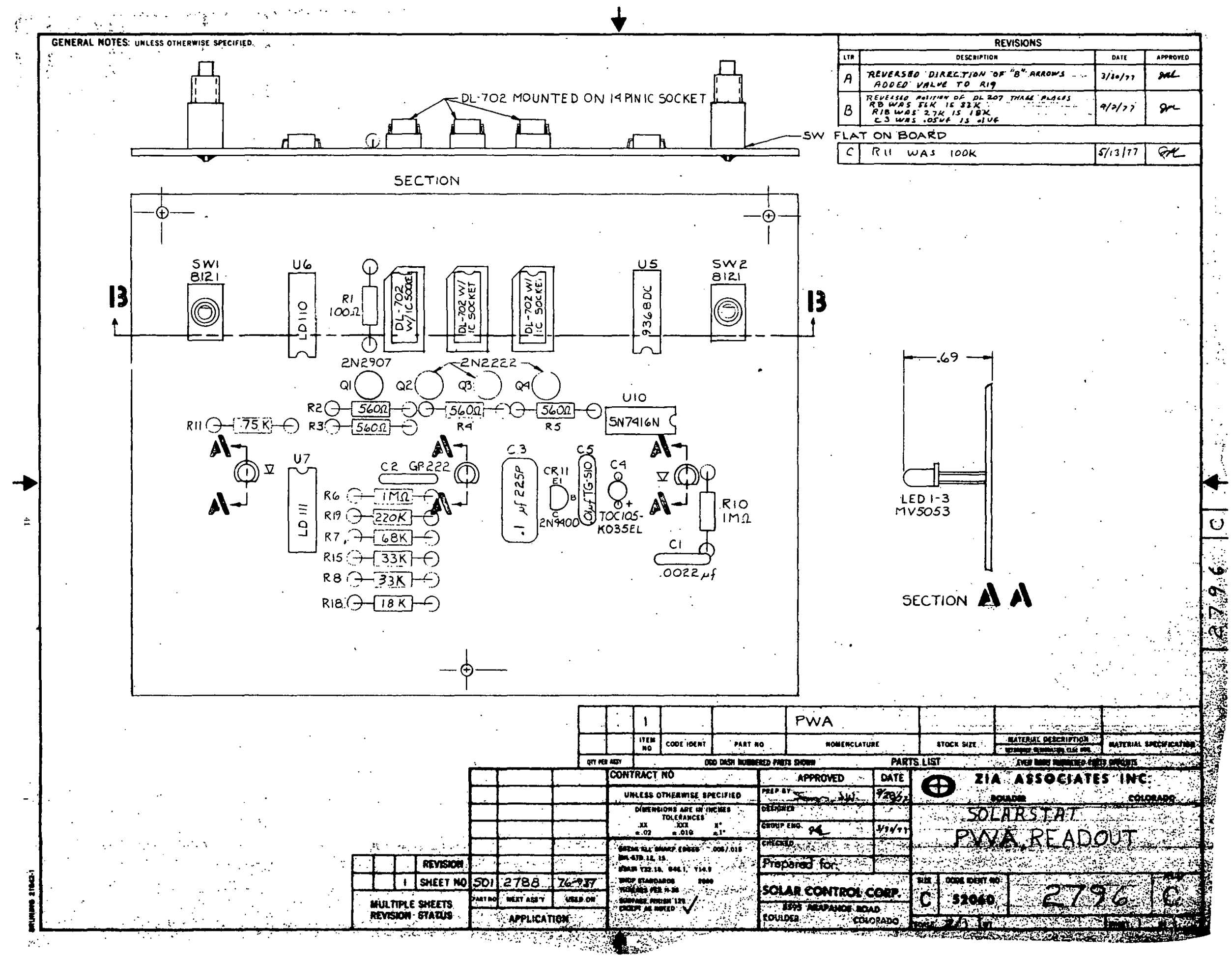




\begin{tabular}{|c|c|c|c|c|c|c|}
\hline \multicolumn{3}{|c|}{ APPLICATION } & \multicolumn{4}{|c|}{ REVISIONS } \\
\hline \multirow[t]{2}{*}{ PMiniso } & NEXT ASSY & USED ON & LTR & DESCRIPTION & DATE & $a$ \\
\hline & & & $A$ & AOOED DESLRIATION TO RIG & $3: 2010$ & \\
\hline & & & & $\begin{array}{l}\text { DELETL CR \&, CA } 9, C R 10 \\
\text { AOO R29,R3 O, R3I } 3.3 \mathrm{~K}\end{array}$ & & \\
\hline & & & & CHANGE RRO FRON 2742 to B2 $\Omega$ & & \\
\hline & & & $B$ & CHANGC R26,27,28 FNodP $720 \times R 1 K, 70 . \therefore$ & & \\
\hline & & & & $\begin{array}{l}R 18 \text { WAS } 27 K \text { is } 18 K \\
R 8 \text { WAS } 8 E K \text { is 33K }\end{array}$ & & \\
\hline & & & $D$ & $\begin{array}{l}\text { CHANSE R2L To } 15 \times 2 \text { Add R32 } \\
\text { CHANSERT }\end{array}$ & & \\
\hline & & & $C$ & 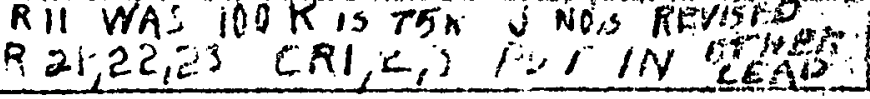 & & of of \\
\hline
\end{tabular}

Properst for:

50LAP Conger conp

II" $55 \% 5$ ARIABATIOE ROAD

BOULOER - COLCRADO

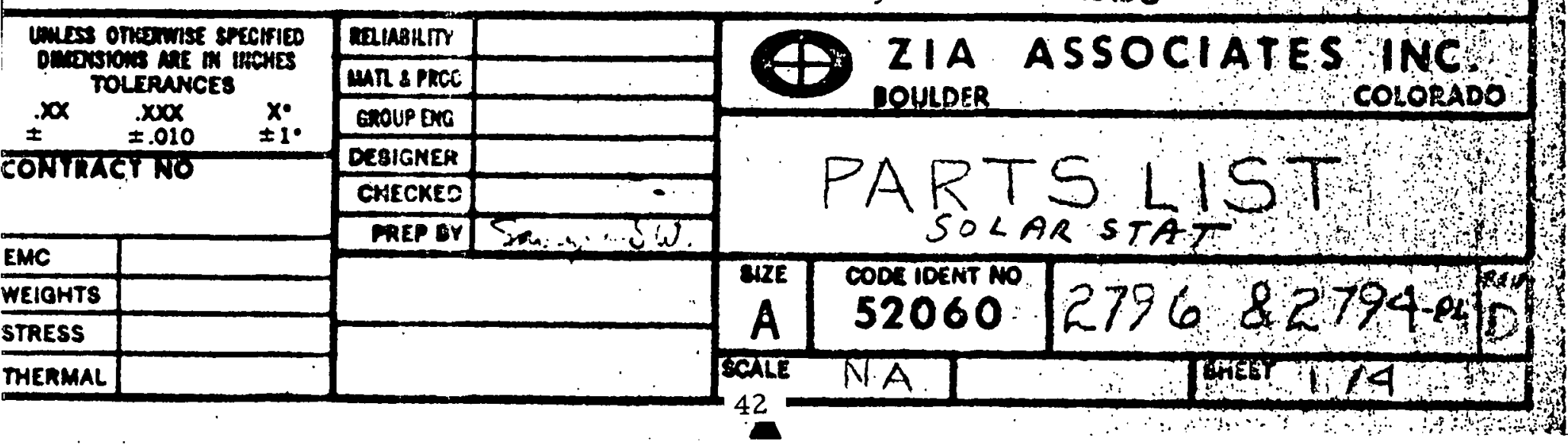




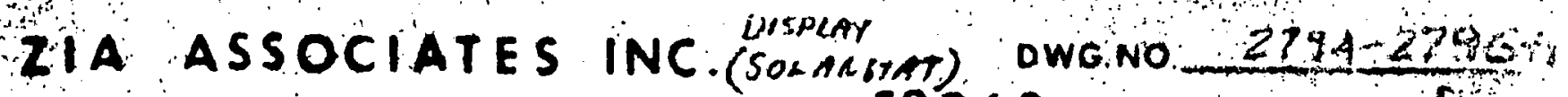
solider colorado code dent no. 520.60 sheet 2 loge rev 1 .

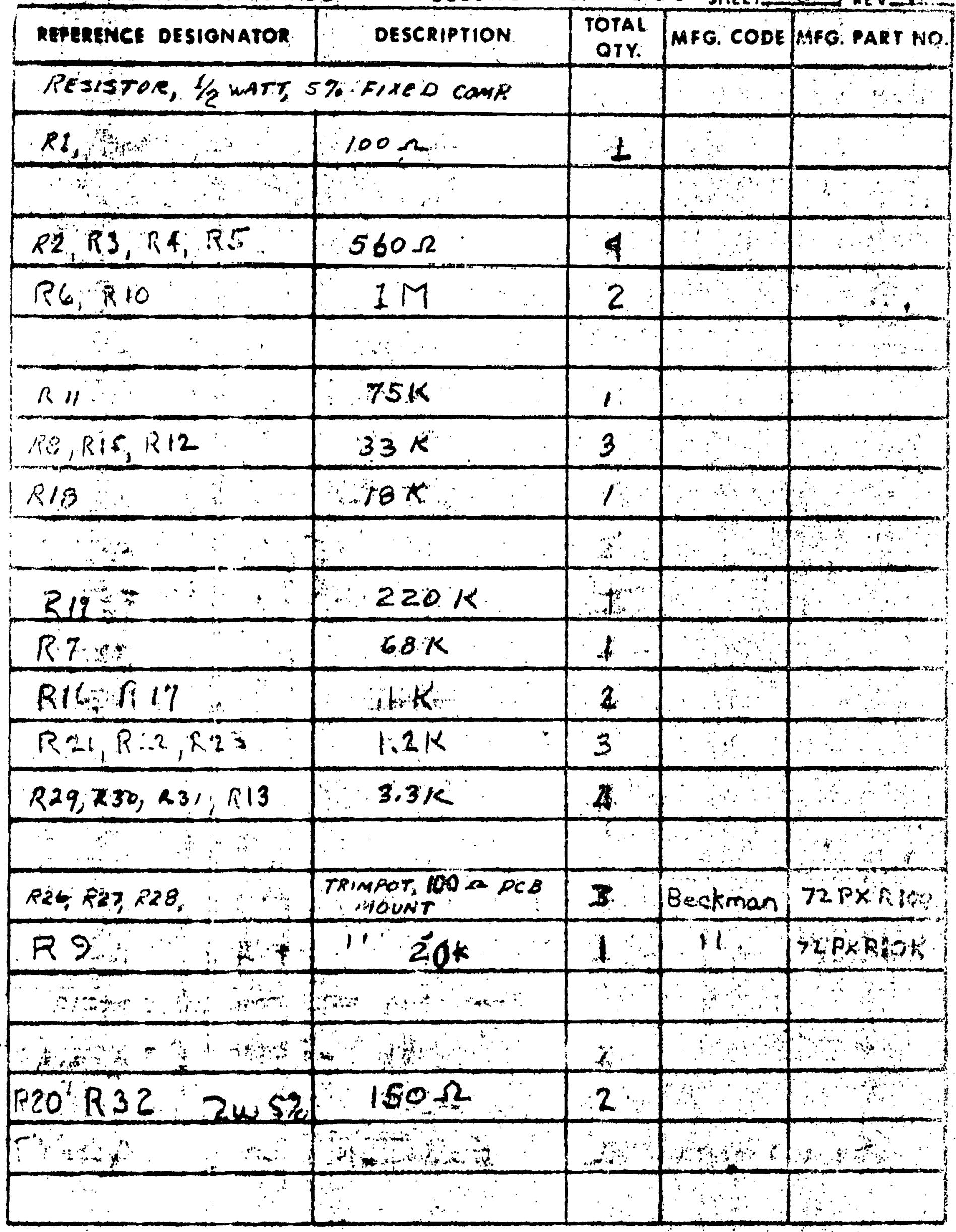

$76-937$

43 


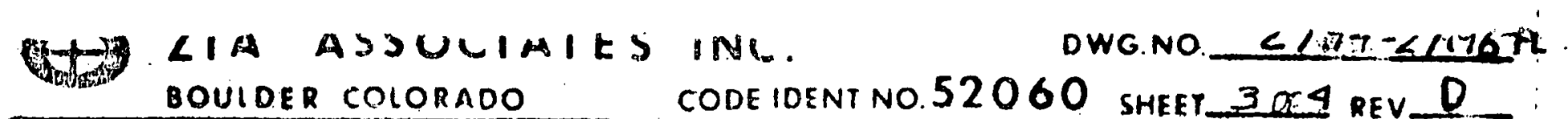

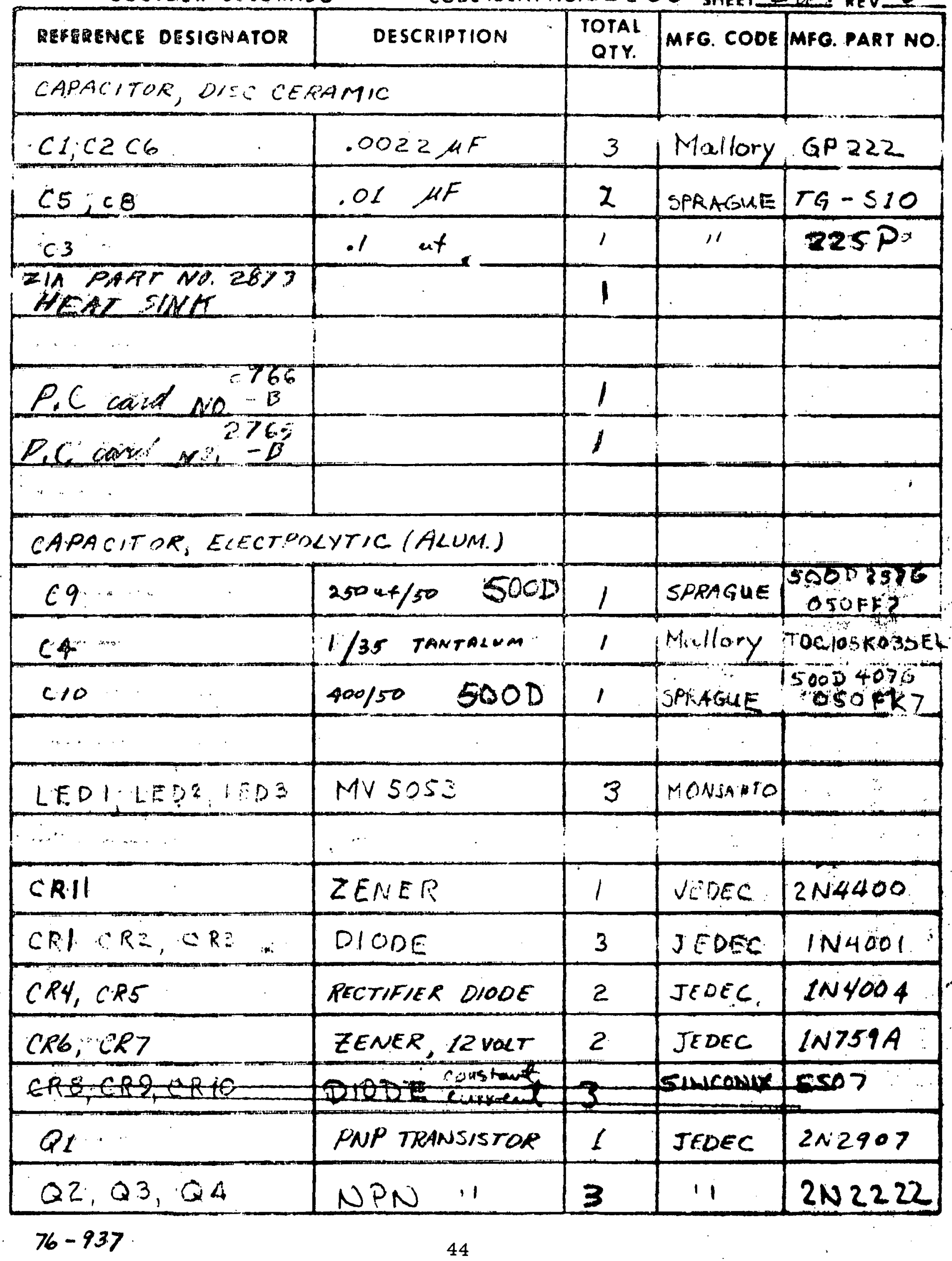


BuZIA ASSOCIATES INC.

DWGNO 27Y\$-27\$6.P1 CODE IDENT NO. 52060 SHEET QCOEZ REV_R

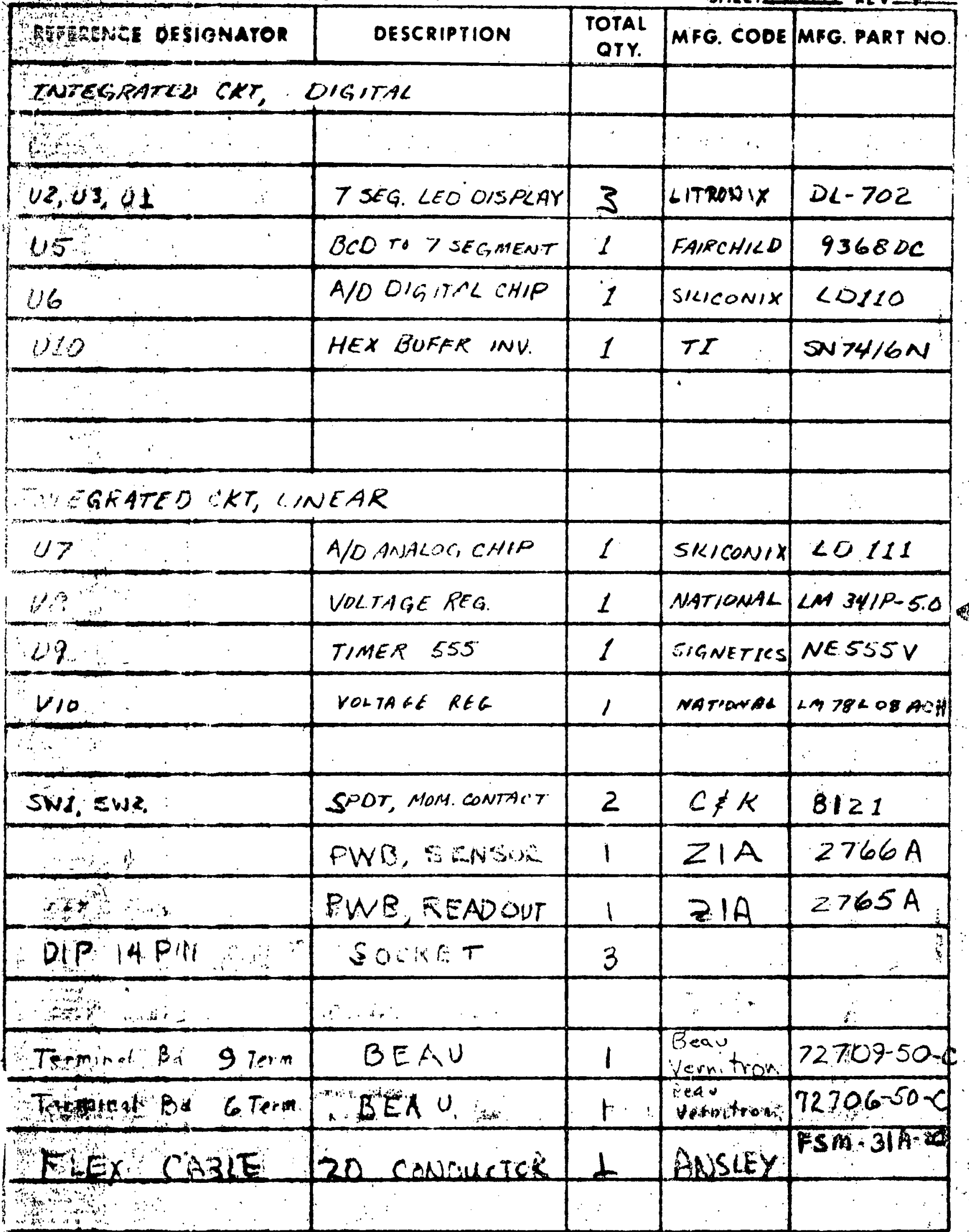


1 IDEAL INDUSTRIES, INC., SYCAMORE, ILL!NOIS.

(2] POT DIODE ASSY. INTO HOUSING WITH CASTALL 301 RESIN. CURE WITH RTT CATALYST FOR 24 HRS. AT $25^{\circ} \mathrm{C}$

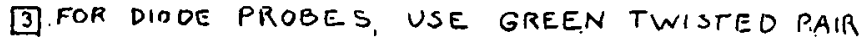
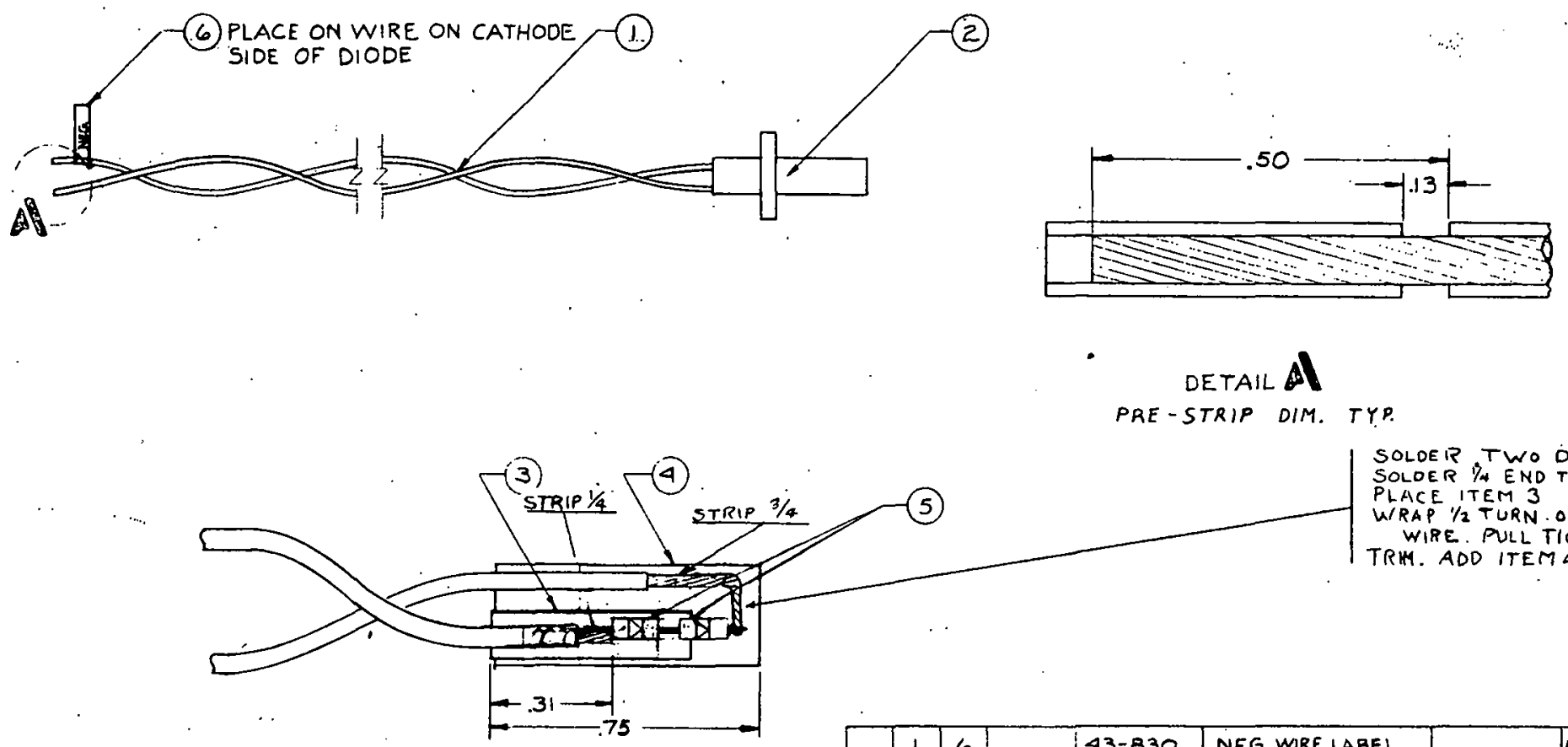

(5)

DETAIL A

PRE-STRIP DIM. TYP.

WIRING DETAIL

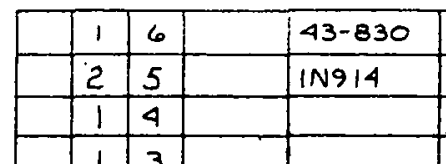

NEG. WIRE LABEL SIGNAL DIODE HEAT SHRINK HEAT SHRINK \begin{tabular}{lll|l|l}
\hline 1 & 3 & & & HEAT SHRIN \\
\hline 1 & 2 & & $2763-1$ & HOUSING
\end{tabular} WVITED FAIR

SOLDER TWO DIODES TOGETHER SOLOER TA END TO BACK DIODE WRAP $1 / 2$ TURN. OF $3 \%$ STRIPPED WIR̈E ON DIODE WIRE. PULL TIEHT TO GLASS AND. SOLDER
TRH. ADD ITEMA. SHRINK. POT

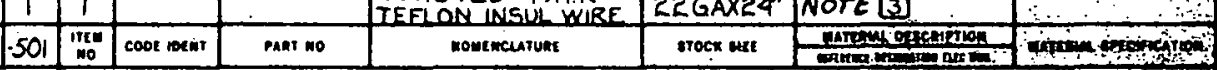

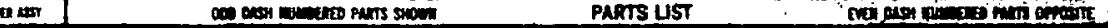

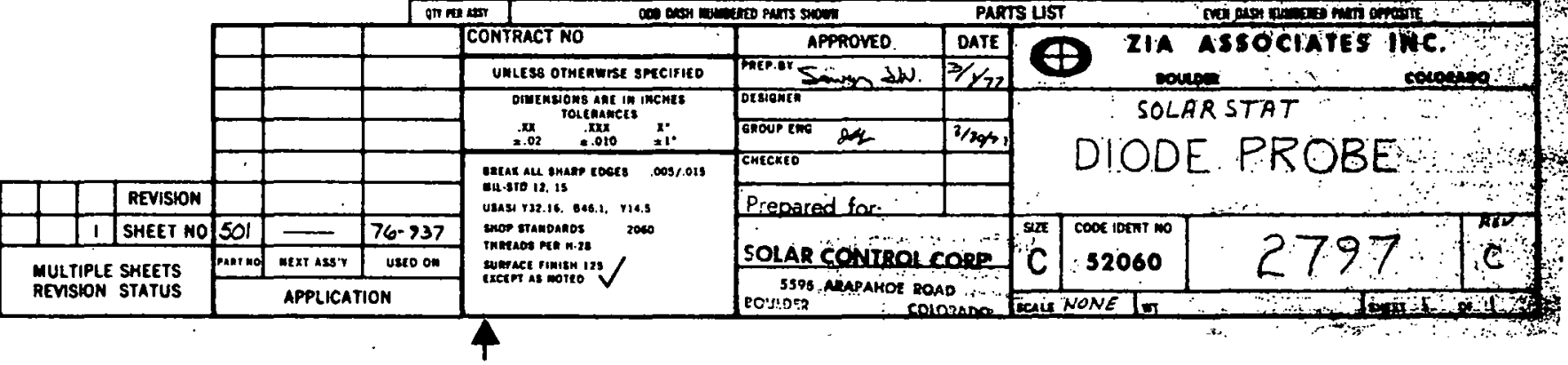




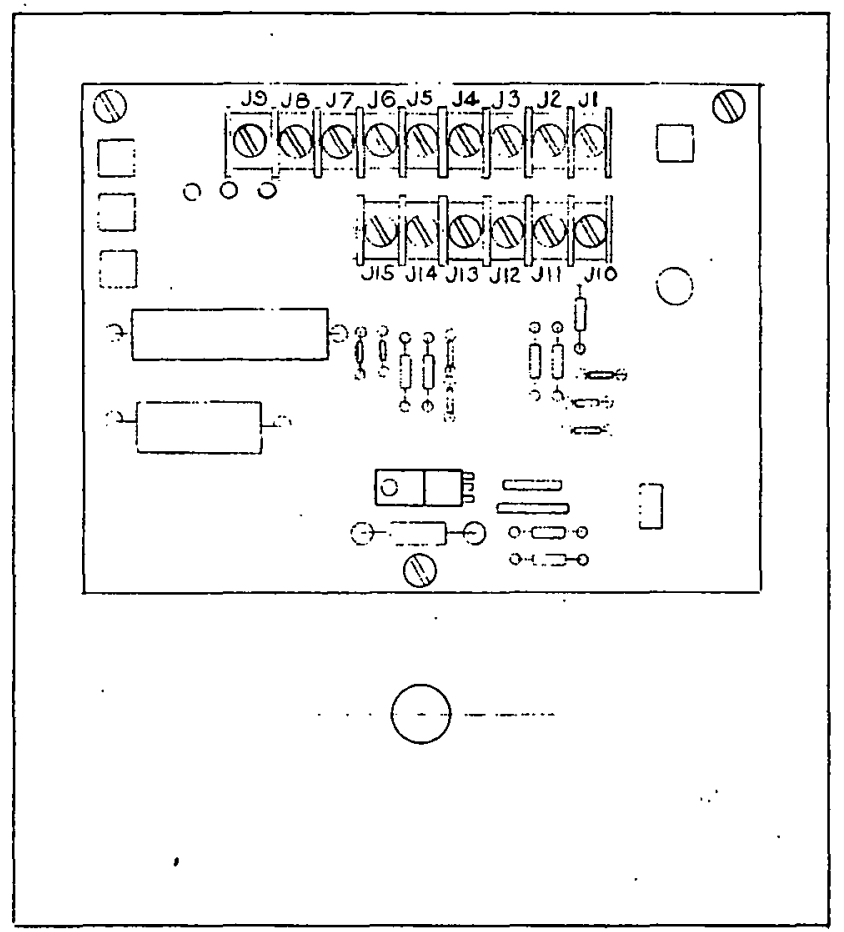

\begin{tabular}{|c|c|}
\hline "J" & INTER FACE \\
\hline 11 & $24 V A C$ \\
\hline 32 & 2.4VAC LO \\
\hline$\sqrt{3}$ & GROUND \\
\hline 34 & STORAGE PROBE \\
\hline$J 5$ & STORAGE PROBE NEG. \\
\hline$\sqrt{ } 6$ & COLLECTO PRDBE \\
\hline 37 & COLLECTOR. PROBE NEG \\
\hline J8 & NORMAL \\
\hline J9 & NORMAL PROBE NEG \\
\hline$J 10$ & $\begin{array}{l}\text { SOLAR COLLECTING } \\
\text { RELAY-2AVAC OR POS RE }\end{array}$ \\
\hline 311 & $\begin{array}{l}\text { SOLAE COLLECTINC } \\
\text { RELAY }\end{array}$ \\
\hline$J 12$ & $\begin{array}{l}\text { SOLAR HEATTN } \\
\text { DELAY DAV AE }\end{array}$ \\
\hline$\sqrt{13}$ & $\begin{array}{l}\text { SOLAR HEATING } \\
\text { RELAY-Z YYACOKS }\end{array}$ \\
\hline J14 & $\begin{array}{l}\text { CONVENTIDNAI HEATING } \\
\text { RELAY-C 4VAC OR POSOC }\end{array}$ \\
\hline J15 & $\begin{array}{l}\text { CONVENTIONAL HEATING } \\
\text { RELAY-Z LYAC OB NEE QC }\end{array}$ \\
\hline
\end{tabular}

* Rec.ommended for outdocr temperature

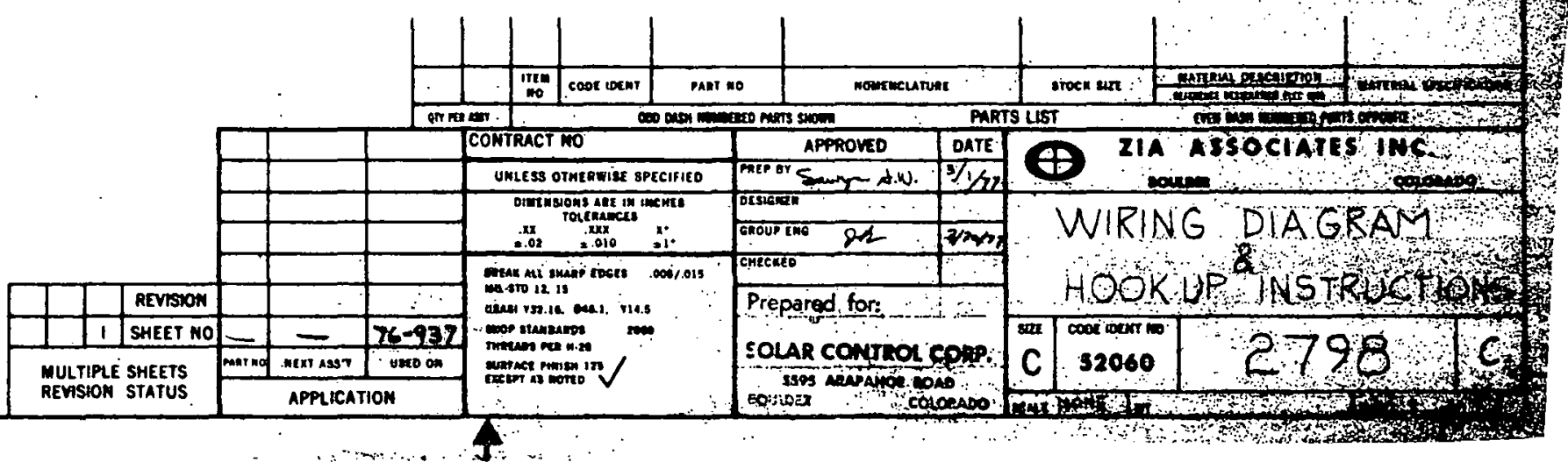




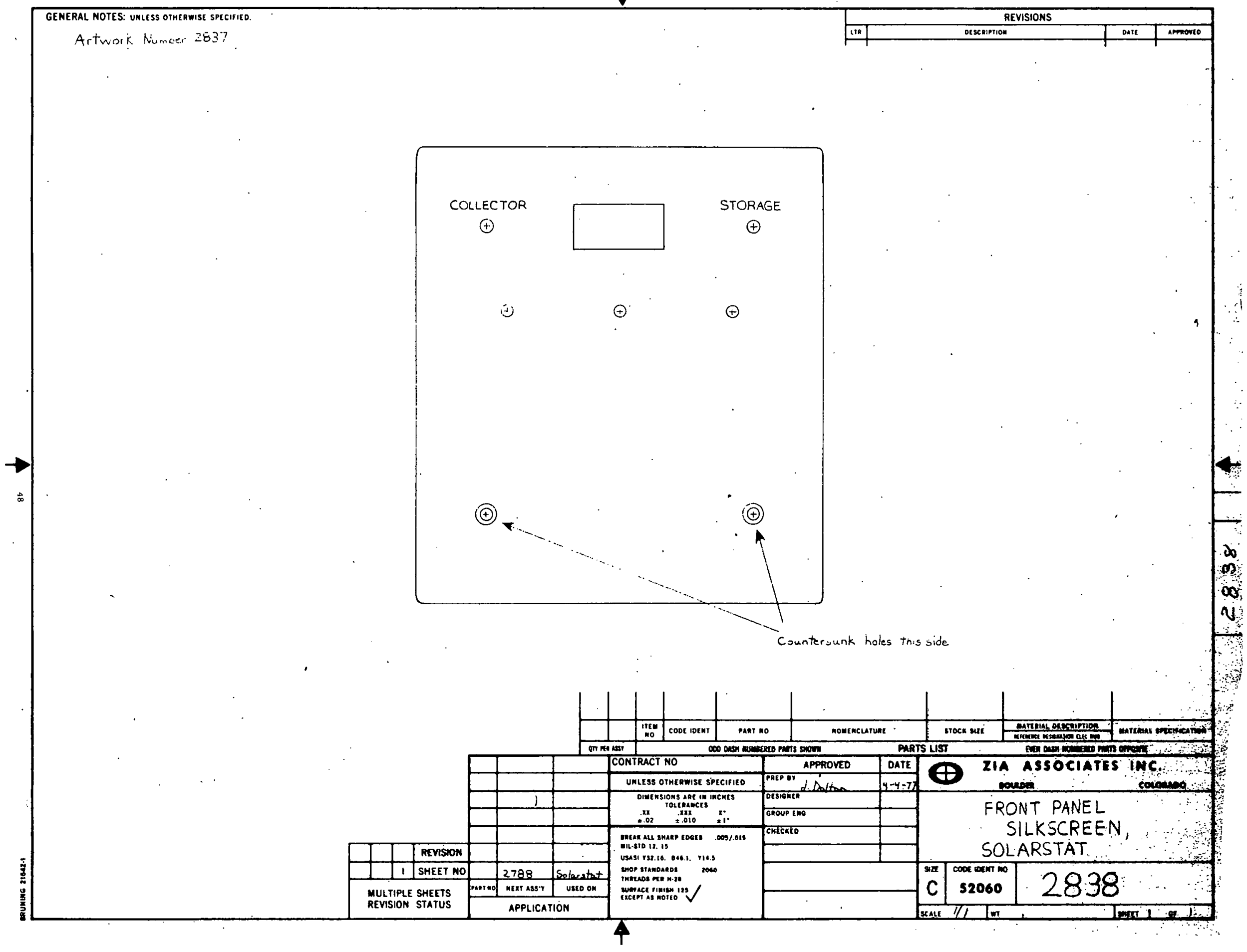




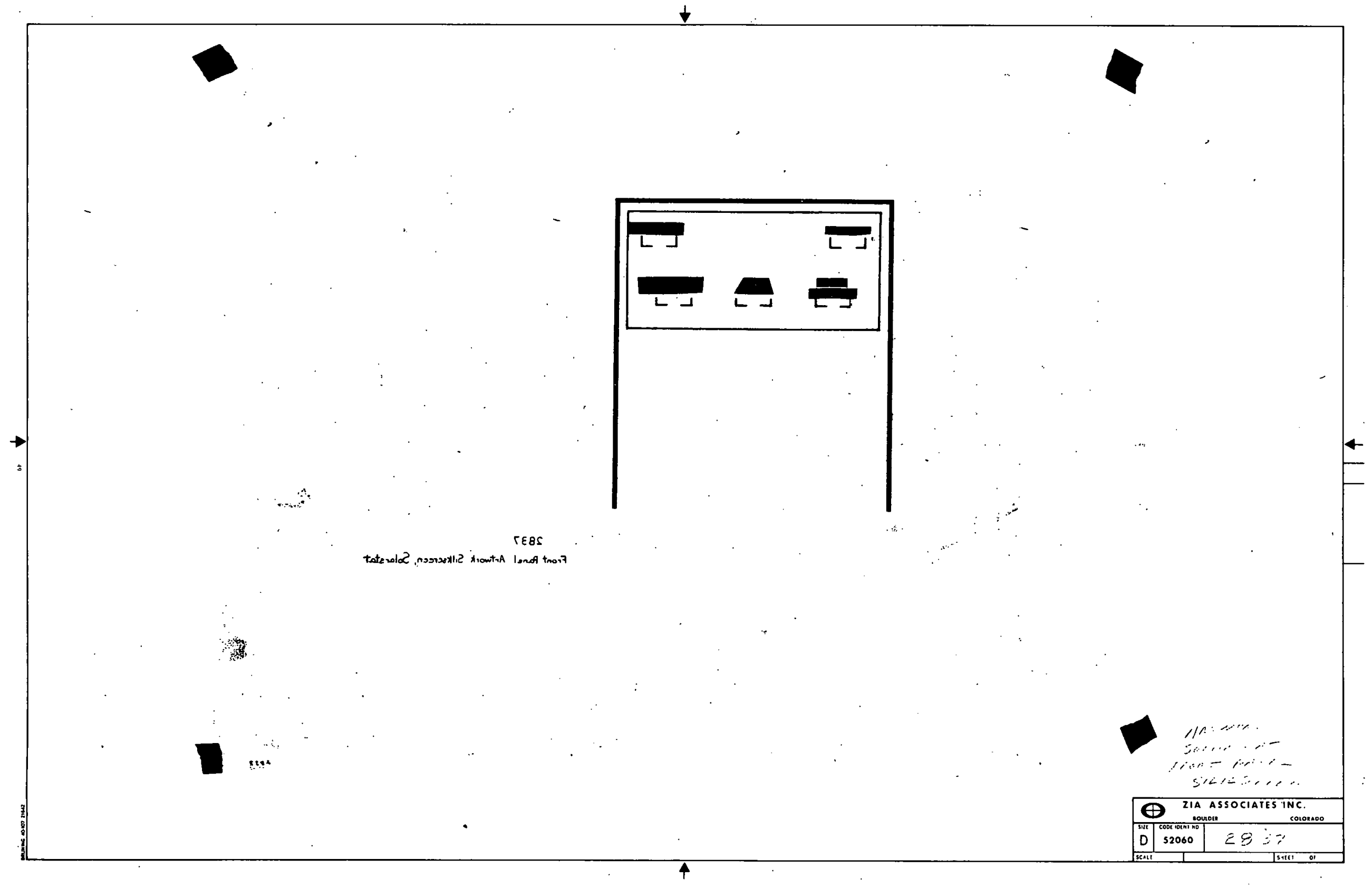




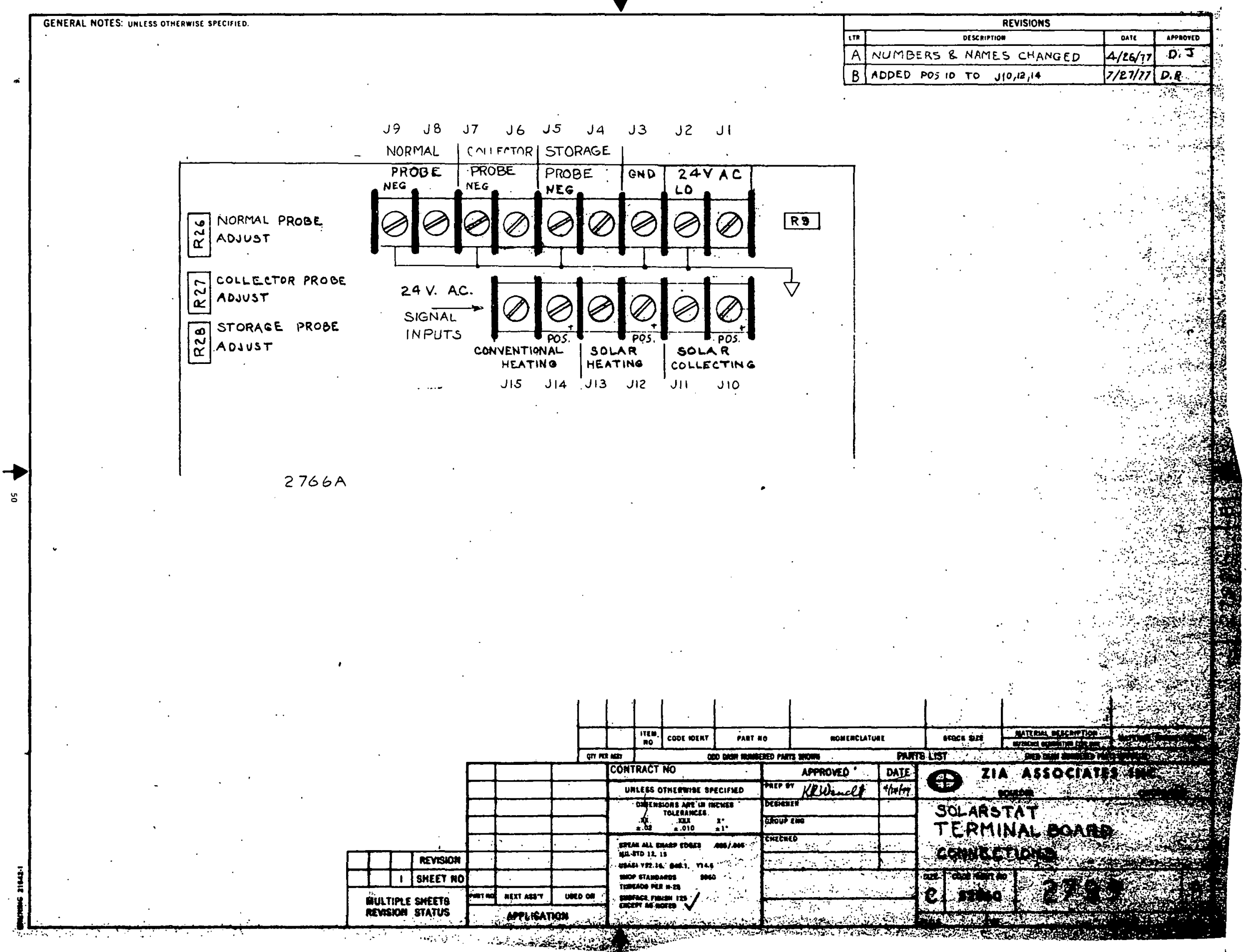




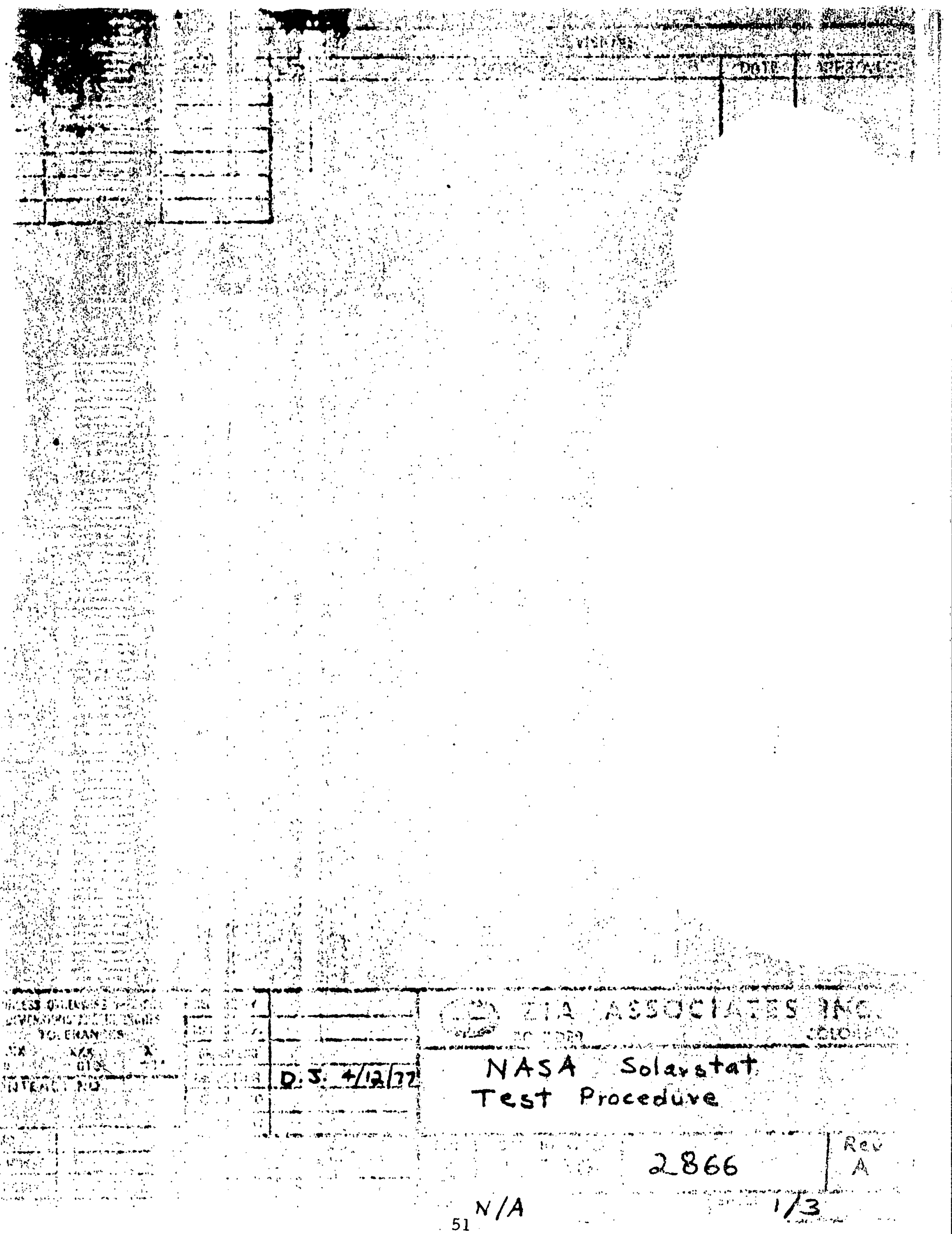




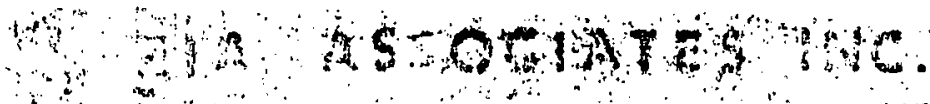

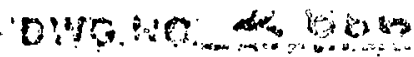

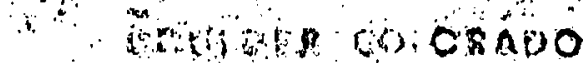
Coverorrine 52060

\section{SOLARSTAT}

Test procoduso

\section{Enuigenent Neiglad}

A. 3i Digit Digital Voltmeter ( \pm Toccuracy)

Q. 24 volt at 1 amp trangformer

C. Thres dual diods temperature probas

D. Aprisoximarely ore quart fomatar bath $\left(32^{\circ} \mathrm{F}\right)$

E. Aphroximatoly one quart not water bath $\left(150^{\circ} 5\right)$

F. $0^{0}$ to $200^{-2} \mathrm{~F}$ thermometer ( \pm zid acruracy)

\section{Progedure}

A. Connect the 24 volt transformor secondary leads to the termlint li:luck screws J1 (Hi) and J2 (ground). (Refer to orswing Pimber 2799 for terminel locations.)

1. Comar the thre temparature probes to the terminal blook screus 54 and $35, J 6$ and $J 7$, and 38 and 29.

c. Contar tho throo vapiatio resistors on the bottom printed circult mard.

b. Connect the nagativa (-) (blk) Digitil. voltmeter lagd to the regative (-) sdde of the 400 micro farad capacitot $\left(c_{10}\right)$.

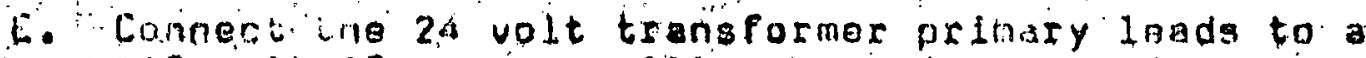
910 volt-ac source. Allow two minutos pefore procaeding.

F. Using tho Digtal voltmeter positiva (+) (rod) laad verify tr.o folinuing voltagoas (reper to Jrawing nuniter 2794 A Pir colajorant location on aensor (lower) board, and $2795 \mathrm{C}$ for tocia ione on the roadout (upper) unard)

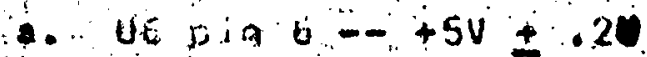

4. UE fin $19--120 \pm 1.2 \mathrm{~V}$

c. Ui ? $1 E-12 \mathrm{E} \pm 1.2 \mathrm{~V}$

d. $170 \sin 15-1.44 \pm .07 \mathrm{~V}$

b. uipin $10-m+a v \pm 3 v$ 
H ASOCATES INC.

G. Remove the Digital volumeter connoction.

H. Place the throe tompraturo probes and thermomotor in tho les watcr bath. Alloy five minutes boforo:proceding.

Varify $32^{\circ}$ r on the thermometer.

While ubserving the LED tomperature display adjust R for diepiey of $32 \%$.

Whil nresing tha left hand push button adjust R27 par a display of $32 \mathrm{~F}$.

i. While presaing the right hand push button adjust 220 for a displas of $32^{\circ}$.

M. Tranfires thes threa conperature probes and thermometer to the hot werer bath. Allow flue minutes bopore oceading.

N. Note ine thermometer reading.

o. Adjuet rg por a temperature display equal to the thermometer rastir.7.

p. Verify the sane display reading ( $t$ l degree) when the lopt and rigiti nand push buttons ate prasegd.

Q. Repant gtops $H$ through $p$ until all three temperature displays match the tnermometer readings for the ice water bath and hot watgr: bath \pm 1 degree.

R. Jumisla the $24 \mathrm{~V}$ from 31 and 32 to 310 and 311 . The oolar colbisicing l.ED hould light. (Left) Jumper $J 1$ and 22 to jis ind Jiz. The solar heating (center) LEO onould light. Finaliy, jumper 31 ho $J 2$ to 314 and 315 . The convontionel nooting (rjolit). LED should ligrit. 


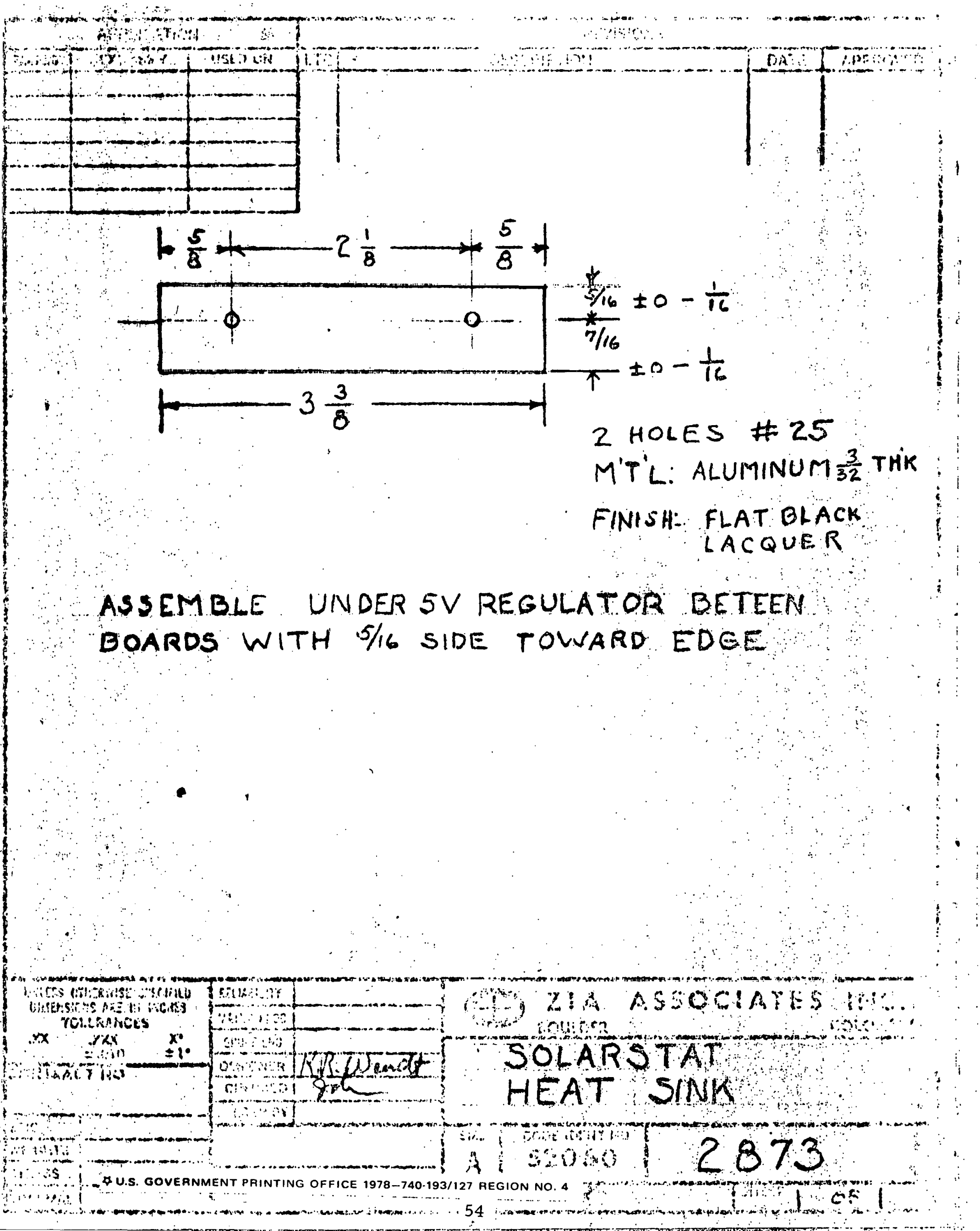

